\title{
On the shelf resonances of the Gulf of Carpentaria and the Arafura Sea
}

\author{
D. J. Webb \\ National Oceanography Centre, Southampton SO14 3ZH, UK \\ Correspondence to: D. J. Webb (djw@ soton.ac.uk) \\ Received: 20 December 2011 - Published in Ocean Sci. Discuss.: 3 February 2012 \\ Revised: 29 June 2012 - Accepted: 6 July 2012 - Published: 1 September 2012
}

\begin{abstract}
A numerical model is used to investigate the resonances of the Gulf of Carpentaria and the Arafura Sea, and the additional insights that come from extending the analysis into the complex angular velocity plane. When the model is forced at the shelf edge with physically realistic real values of the angular velocity, the response functions at points within the region show maxima and other behaviour which imply that resonances are involved but provide little additional information. The study is then extended to complex angular velocities, and the results then show a clear pattern of gravity wave and Rossby wave like resonances. The properties of the resonances are investigated and used to reinterpret the response at real values of angular velocity. It is found that in some regions the response is dominated by modes trapped between the shelf edge and the coast or between opposing coastlines. In other regions the resonances show cooperative behaviour, possibly indicating the importance of other physical processes.
\end{abstract}

\section{Introduction}

Observational studies of the tides and the success of the standard method of tidal prediction show that although non-linear effects are present, the response of the ocean to tidal forcing is predominantly linear. In terms of understanding ocean physics, this is important because it means that we can make use of the theory of linear operators. Such an approach has been very successful in the development of many other areas of physics.

One of the theory's main results is that the response of linear systems can be expressed in terms of a Green's function (Webb, 1973b), and that this is an analytic function of an- gular velocity with poles at the eigenvalues or resonances of the system. The response to a particular forcing, the response function, has similar properties. The residue at its poles has a spacial structure proportional to the corresponding eigenfunction and an absolute magnitude depending on how well the mode is excited by the forcing.

If the system is frictionless, the poles lie on the real axis, but if friction is present, they move off the real axis into the negative imaginary half of the complex plane. The distance from the real axis then equals the decay rate of the resonances.

The theory was used by Zahel and Müller (2005) and Müller (2007) to investigate the resonances of the global ocean. Their results using a one degree model show that between the diurnal and semi-diurnal tides the resonances have a separation of about 0.3 radians per day ${ }^{1}$, and that the imaginary components range between -0.13 and -0.6 radians per day with an average around -0.3 radians per day, corresponding to a decay time of about three days.

This decay time, which corresponds to an energy decay time of $36 \mathrm{~h}$, is slightly longer than the observational evidence which indicates an energy decay time of less than $30 \mathrm{~h}$ (Miller, 1966; Garrett and Munk, 1971; Webb, 1973a; Egbert and Ray, 2003). However, although a resolution of one degree is sufficient to resolve many features of the deep ocean basins, where tidal wavelengths are large, it is insufficient for many of the details of continental shelves, where the wavelengths are much shorter.

\footnotetext{
${ }^{1}$ To make the connection with the tides clearer this paper uses the "practical" units of radians per day. In these units the diurnal tides lie near $2 \pi$ radians per day and the semi-diurnal tides near $4 \pi$ radians per day.
} 
This is critical because a large fraction of the energy of the tides is lost on continental shelves, especially those which are about a quarter of a wavelength wide. Webb (1976) used a simplified model to show that quarter wavelength resonances are very effective at coupling such shallow shelves to the deep ocean. Other studies using analytic and simplified numerical models include Webb (1976, Appendix 2), Webb (1982), Arbic et al. (2009) and Arbic and Garrett (2010).

Observational evidence indicates that the English Channel, Hudson Strait, the Argentinian Shelf and the NW Australian Shelf are all regions where there are both significant amounts of tidal dissipation and quarter wavelength resonances. Zahel and Müller (2005) show energy being lost around the British isles and on the Argentinian shelf but very little being lost in the other two regions. This is probably because the model does not fully represent the geometry of such regions.

If this is true, then the shallow continental shelves will contribute extra modes to the tidal bands. The result will be a greater density of modes and more overlapping modes than was found in their model.

Other studies have investigated the effect of resonances on the ocean but with timestepping models limited to real values of angular velocity. Griffiths and Peltier (2008, 2009) have investigated the effect of changes in sea level on a global ocean and have shown that both the Arctic Ocean and Antarctic coastline may become resonant during an ice age. A related study by Green (2010) concluded that increased tidal amplitudes at the last glacial maximum was due to reduced damping of the deep ocean resonances.

The effects of resonances of a global model were also investigated by Arbic et al. (2009) in a study stimulated by the results of a simple model coupling a shelf mode with one in the deep ocean. They found, as did Webb (1976), that even when the angular velocities match, the coupling is strong only when the impedance of the shelf matches the impedance of the deep ocean.

There have also been many studies of known continental shelf resonances using limited area models. Early ones include the study by Duff (1970) of the Bay of Fundy and Fong and Heaps (1978) study of the Bristol Channel. More recently, Arbic et al. (2007) investigated the shelf resonances of Hudson Strait and Ungava Bay using a time stepping model. They first estimated the position of a resonance by fitting a simple pole to the solution calculated at three nearby real angular velocities. A more accurate estimate was then obtained by letting this solution freely oscillate and decay.

The present paper takes a different approach to these papers, in that its main concern is with the overall structure of the response function as a function of complex angular velocity. It concentrates on a realistic section of continental shelf and uses the theory of linear operators to calculate the eigenvalues and eigenfunctions of each resonance and to investigate how they combine to generate the actual response at real values of angular velocity.
However, before doing this the paper first investigates what can be learnt by limiting the calculations themselves to real angular velocities. When this is done, the responses at different locations show evidence of shelf resonances, but the resonance contributions overlap so that it is difficult to extract quantitative information from the data or to obtain any additional insights.

In contrast, when the calculations are extended to complex angular velocities, the resulting three-dimensional figures show a rich pattern of resonances in the neighbourhood of the tidal bands. The analytic properties of the response function can then be used to accurately determine the resonance eigenvalues and eigenfunctions, and the results used to learn about their physical properties and to study how they interact.

The numerical model used is a linear one similar to that of Zahel and Müller (2005). The justification for making such a choice is that, as mentioned previously, almost everywhere in the world the non-linear terms in the barotropic tide, due to friction, inertia and internal tides, are small. In addition, any discussion of resonances is really only valid for physical systems that can be treated as approximately linear.

The area of study chosen covers the Gulf of Carpentaria and the Arafura Sea. This has been done because, firstly, it enables the use of a model which has been validated in a study of both the diurnal and semi-diurnal tides of the region (Webb, 1981). Thus the model should give good results over the whole band of frequencies around one and two cycles per day.

Secondly, it is a large continental shelf. It therefore might be expected to show many of the resonance features to be found elsewhere. Previously Buchwald and Williams (1975) investigated the resonances of the region using a simpler model, and it is also of interest to compare results from the two types of model.

Thirdly, related to point two, tidal observations indicate that the northern Arafura Sea supports both quarter and threequarter wavelength resonances. Tidal heights are smaller than those found in the classic resonance regions, such as the Bay of Fundy and the Bristol Channel, but it does provide an opportunity to learn more about such resonances.

Finally, the original model code (Webb, 1981) is not limited to studies with real angular velocities but, like that used by Zahel and Müller (2005), allows a full investigation of the complex angular velocity plane. ${ }^{2}$.

The results reported here show that by extending the analysis to complex values of angular velocity, it is possible to obtain new insights and obtain a better understanding of the way resonances influence the tides. Such an approach provides a more accurate description of the spatial structure of

\footnotetext{
${ }^{2}$ The present model was used in a similar way by Grignon (2005) to study a simplified representation of the English Channel. It is hoped that a follow up study using a more realistic model of the region will be published soon.
} 
each resonance and makes it easier to separate off the effect of nearby resonances in the overall response of the ocean.

\section{The numerical model}

A numerical model is used to solve Laplace's tidal equations. In vector notation and with a linear friction term these are:

$$
\begin{aligned}
\partial \boldsymbol{u} / \partial t+\boldsymbol{f} \times \boldsymbol{u}+(\kappa / h) \boldsymbol{u}+g \nabla \zeta & =g \nabla \zeta_{\mathrm{eq}}, \\
\partial \zeta / \partial t+\nabla \cdot(h \boldsymbol{u}) & =0 .
\end{aligned}
$$

$\boldsymbol{u}$ is the depth averaged horizontal velocity, $t$ is time, $\zeta$ the sea level, $\zeta_{\text {eq }}$ the height of the equilibrium tide (corrected for Earth tides), $g$ the acceleration due to gravity, $\kappa$ the linear friction coefficient, $h$ the depth, and " $\times$ " indicates a vector product. The Coriolis vector $\boldsymbol{f}$ is defined by:

$\boldsymbol{f}=2 \Omega \cos (\theta) \boldsymbol{n}_{z}$,

where $\Omega$ is the Earth's rotation rate, $\theta$ the co-latitude, and $\boldsymbol{n}_{z}$ the unit vertical vector. The equations are obtained by integrating the full equations of motion in the vertical and neglecting the vertical acceleration, non-linear and selfattraction terms.

Equation (1) is linear, so the general solution for a given forcing can be written as a linear combination of solutions of the form:

$\left(\begin{array}{l}\boldsymbol{u}(t) \\ \zeta(t)\end{array}\right)=\Re\left[\left(\begin{array}{l}\boldsymbol{u} \\ \zeta\end{array}\right) \exp (-i \omega t)\right]$

where $\omega$ is the angular velocity, and $\Re$ represents the real part of the complex expression.

If we define $P$ and $Q$ as

$P=g(i \omega-\kappa / h) /\left[(i \omega-\kappa / h)^{2}+f^{2}\right]$,

$\boldsymbol{Q}=\boldsymbol{f} /\left[(i \omega-\kappa / h)^{2}+f^{2}\right]$,

then

$\boldsymbol{u}=(P+\boldsymbol{Q} \times) \nabla \zeta^{\prime}$,

where

$\zeta^{\prime}=\zeta-\zeta_{\text {eq }}$.

Substituting for $\boldsymbol{u}$ in Eq. (1),

$$
\begin{aligned}
h P \nabla^{2} \zeta^{\prime}+[\nabla(h P)+\nabla \times(h \boldsymbol{Q})] \cdot \nabla \zeta_{1}^{\prime} & \\
& -i \omega \zeta^{\prime}=-i \omega \zeta_{\text {eq }} .
\end{aligned}
$$

This is the equation that is solved numerically in the model.

At coastlines the normal component of velocity is zero as the model depth there is required to be non-zero. If $\boldsymbol{n}_{\mathbf{c}}$ is the unit vector normal to the coast, then from Eq. (5):

$(P+\boldsymbol{Q} \times) \nabla \zeta^{\prime} \cdot \boldsymbol{n}_{\mathbf{c}}=0$.

On the open boundary the amplitude and phase of the incoming wave needs to be specified. In terms of the classification of differential equations, this corresponds to using Dirichlet boundary conditions.

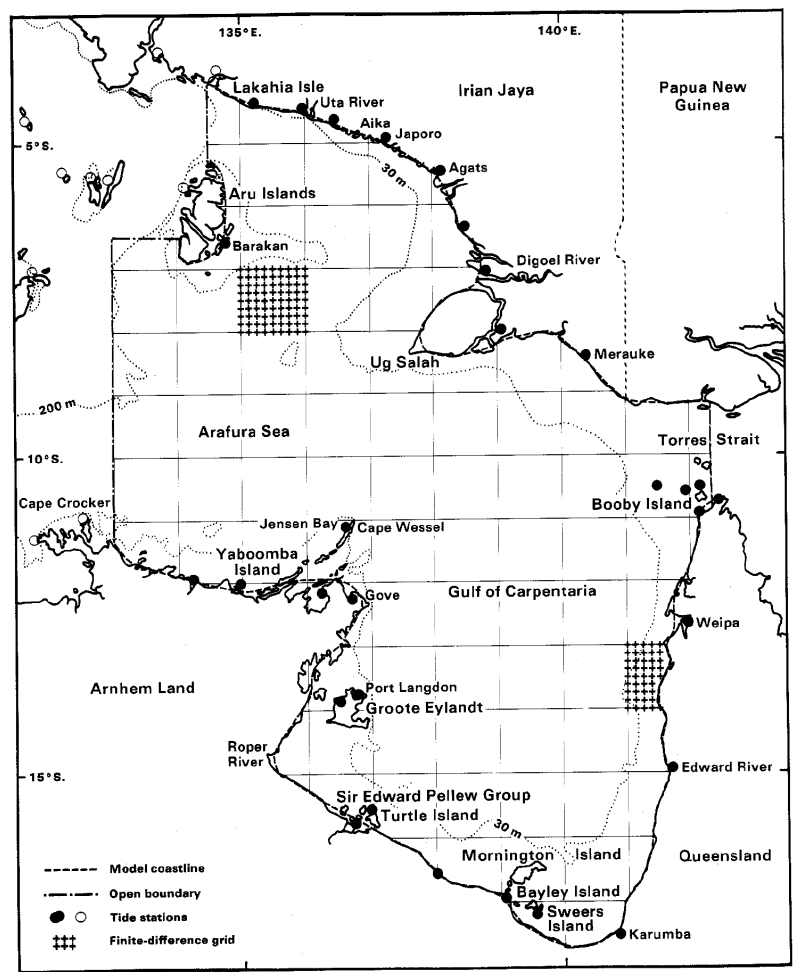

Fig. 1. Plan of the study area, showing the smoothed coastline used in the numerical model. $\circ$ Tidal station used to determine the open boundary conditions. $\bullet$ Other tidal stations. Depth contours are shown as dotted lines. Islands whose coastlines are not represented in the model are replaced by water of depth $2.5 \mathrm{~m}$.

\section{Numerical solution}

In the numerical model, the spherical co-ordinate form of Eq. (7) is replaced by a set of finite difference equations at the vertices of a rectangular grid, using a grid spacing of one eighth of a degree. The boundary condition, Eq. (8), is applied at points where the grid intersects coastlines, the finite difference equations taking into account both the angle between the coastline and the grid and the curvature of the coastline. Both coastline and depths were taken from Admiralty charts of the region.

In the original model (Webb, 1981), observed values of the tidal height were imposed on the open boundaries to the west of the Arafura Sea and on the eastern side of Torres Strait. For the new study reported here, the wave entering the region from the west is assumed to have unit amplitude and constant phase all along the western boundary. In the east, Torres Strait is now blocked.

The use of a constant phase in the west is partly justified by the fact that the phase speed in the deep water along the boundary is large. As a result both the M2 and $\mathrm{K} 1$ tides have, to a first approximation, constant amplitude and phase along the western boundary. Also, as Dirichlet boundary conditions 


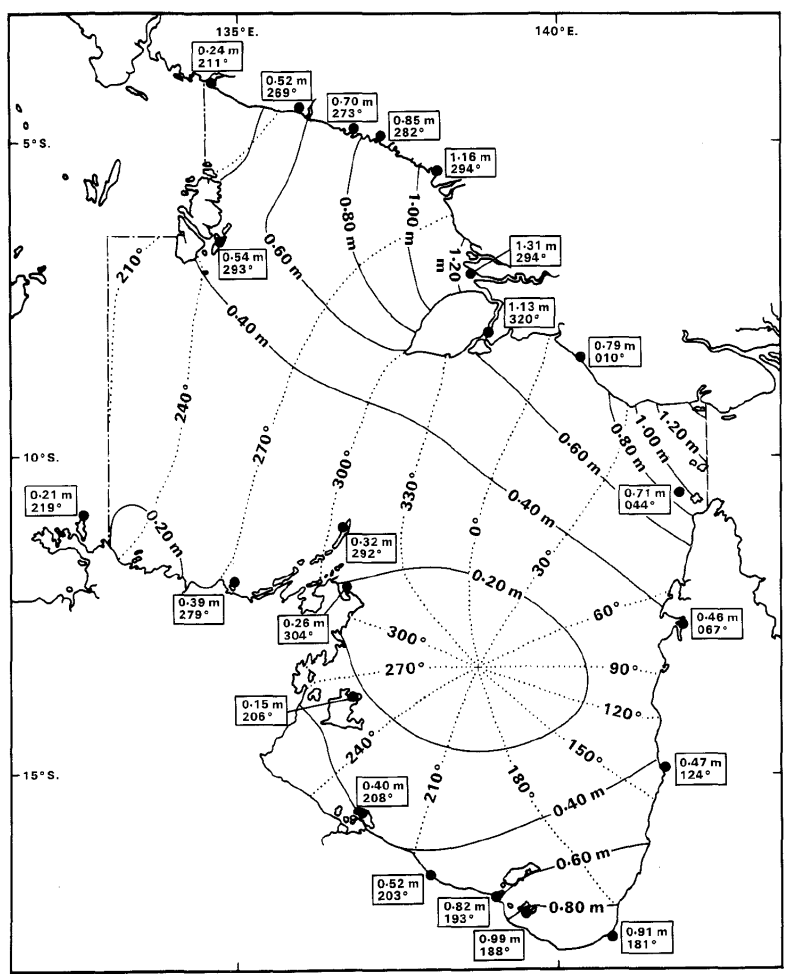

Fig. 2. Computed co-amplitude and co-phase lines for the $\mathrm{K} 1$ tide in the Gulf of Carpentaria and Arafura Sea. Observed amplitudes and phases, shown in the square boxes, are taken from Easton (1988) and from the Admiralty Tide Tables (Anon, 1971).

are used, the resonance eigenvalues and eigenfunctions are independent of the forcing used for the model.

In the east the original tidal study (Webb, 1981) showed that the amount of tidal energy entering through Torres Strait is small and has little effect on the tides within the Gulf. This might be expected given large differences in tidal phase between the opposite ends of the strait (Anon, 1971) and is confirmed by the observations of Wolanski et al. (1988). Thus, the decision to simplify the analysis by blocking Torres Strait should have little effect on the large-scale response of the Gulf region.

The coefficients resulting from the finite difference equations are loaded into a sparse matrix and the associated forcing terms into a corresponding vector. The full matrix equation is then solved using Gaussian elimination and back substitution, the numbering of the vertices being organised to minimise the size of the intermediate matrix. Further details of the model and solution are given in Webb (1981).

\section{The behaviour at tidal frequencies}

Figures 2 and 3, reproduced from Webb (1981), show the original model's solution for the K1 and M2 tides. In the figures, the amplitude and phase lines are from the model and

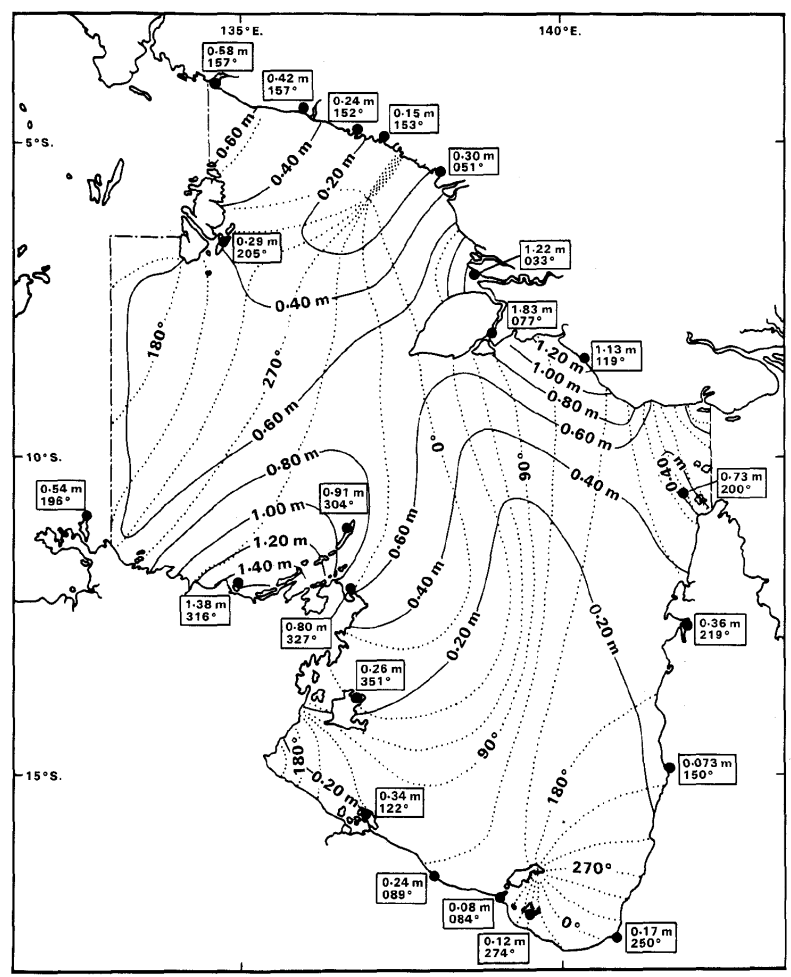

Fig. 3. Computed co-amplitude and co-phase lines for the M2 tide. Observed amplitudes and phases, shown in the square boxes, are taken from Easton (1988) and from the Admiralty Tide Tables (Anon, 1971).

the boxed figures show the same quantities measured at tide gauges. There are discrepancies, but they are relatively small. Wolanski (1993) later analysed the tides of the region, making use of additional current meter data, and found similar tidal patterns. The main difference was for the $\mathrm{K} 1$ tide, where the centre of the amphridrome was located $100 \mathrm{~km}$ further north.

The present model results for the $\mathrm{K} 1$ amplitude and phase lines indicate that, to a first approximation, the diurnal tide propagates along the northern boundary as a Kelvin wave and that it then circles clockwise around the Gulf of Carpentaria losing energy on the way. However, a simple decaying Kelvin wave would have an amplitude which declined monotonically with distance. Instead, the model shows four regions where the amplitude at the coast is a local maximum.

Three of these, in corners of the Gulf may result from the Kelvin wave propagating around a nearly right-angled corner. The fourth, in the north-east of the Arafura Sea, near $138^{\circ} \mathrm{E}, 7^{\circ} \mathrm{S}$, could be associated with a quarter wave resonance between the Digoel River ${ }^{3}$ and the shelf edge.

In contrast, the semi-diurnal M2 tide shows no simple resemblance to a Kelvin wave. In the north of the Arafura Sea, there appears to be a $3 / 4$ wavelength wave, trapped between

\footnotetext{
${ }^{3}$ For locations see Fig. 1.
} 

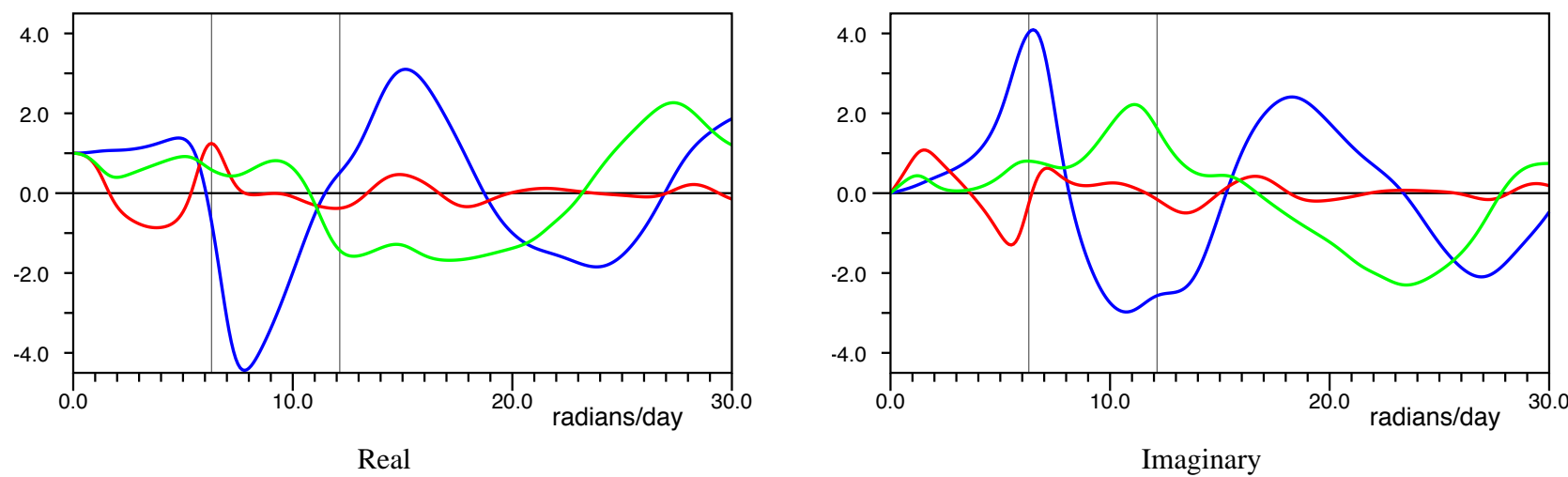

Fig. 4. Real and Imaginary components of the response functions between zero and 30 radians per day for Digoel River (blue), Karumba (red) and Yaboomba Island (green). Vertical lines mark the angular velocities corresponding to the $\mathrm{K} 1$ (6.30 radians day $^{-1}$ ) and $\mathrm{M} 2$ $\left(12.14\right.$ radians day $\left.^{-1}\right)$ tides.

the shelf edge and the Digoel River. There are two additional maxima in the south of the Arafura Sea and the north of the Gulf, which may be related, and a low amplitude east-west oscillation in the centre and south of the Gulf.

These results emphasise the fact that individual tidal constituents give little insight into the physical processes involved. It is possible to repeat the analysis with other tidal constituents, but the tidal bands are so narrow that little more can be learnt.

However, in the present case the model is not limited to the tidal bands. It can be forced with a wide range of frequencies and so provide a wealth of additional information.

\section{The response function}

Using the new boundary conditions, the model was run at a series of frequencies between zero and 30 radians per day ( $\sim 4$ cycles per day). It is impractical to show co-tidal charts for all the frequencies calculated, so instead Figs. 4 to 7 show the response $R$ at three stations which have been selected to illustrate a range of behaviour. $R$ is defined as

$R(x)=\zeta(x) / \zeta_{\mathrm{b}}$,

where $\zeta(x)$ is the (complex) tidal height at position $x$, and $\zeta_{\mathrm{b}}$ is the tidal height on the open boundary.

The first station is near Digoel River in the north of the Arafura Sea where both the diurnal and semi-diurnal tides indicate the presence of standing waves. The second is near Karumba in the south-east of the Gulf of Carpentaria. Resonances do not appear to be a key feature of the region, but there is a strong contrast between the diurnal and semidiurnal tides. The final point is near Yaboomba Island on the southern boundary of the Arafura Sea. The diurnal tide appears as a simple progressive wave along the coast, but the semi-diurnal tide appears to be affected by a resonance.

The results are presented here in three different ways. The first, in terms of the real and imaginary components of $R$, is the most fundamental and is closely related to the later exploration of the complex $\omega$ plane. The second, in terms of the amplitude and phase, is easier to relate to the physics, and this is also true of the third, where the real and imaginary components are plotted against each other.

\subsection{Real and imaginary components}

Figure 4 shows the real and imaginary components of the response function at each station. As the angular velocity tends to zero, all the real values tend to unity and the imaginary values to zero, indicating that the tide everywhere follows that on the open boundary. As the angular velocity increases, the functions diverge, with the Digoel River showing the largest amplitude excursions. At Karumba the components have much smaller excursions, especially at higher angular velocities. Neighbouring maxima and minima are also much closer than for Digoel River.

At Yaboomba Island the oscillations in the real and imaginary components at low angular velocities are less than at the two other stations. However, near 11.5 radians per day there is a change in sign of the real component and a maximum in the imaginary component, after which both components show oscillations comparable with those of Digoel River.

\subsection{Amplitude and phase}

Figure 5 re-plots this data in the form of the amplitude and phase of the response. The amplitudes are all one at zero angular velocity, but at higher values the behaviour is very different. Thus, over most of the range the Digoel River amplitude is above two; it also shows very strong peaks near 7 and 14 radians per day and a weaker broader one near 27 radians per day.

The Karumba amplitude shows a small peak near 1.5 radians per day, a second one near 6 radians per day, but at higher angular velocities the amplitude is much reduced. There is a broad low peak around 13 radians per day and a further one 

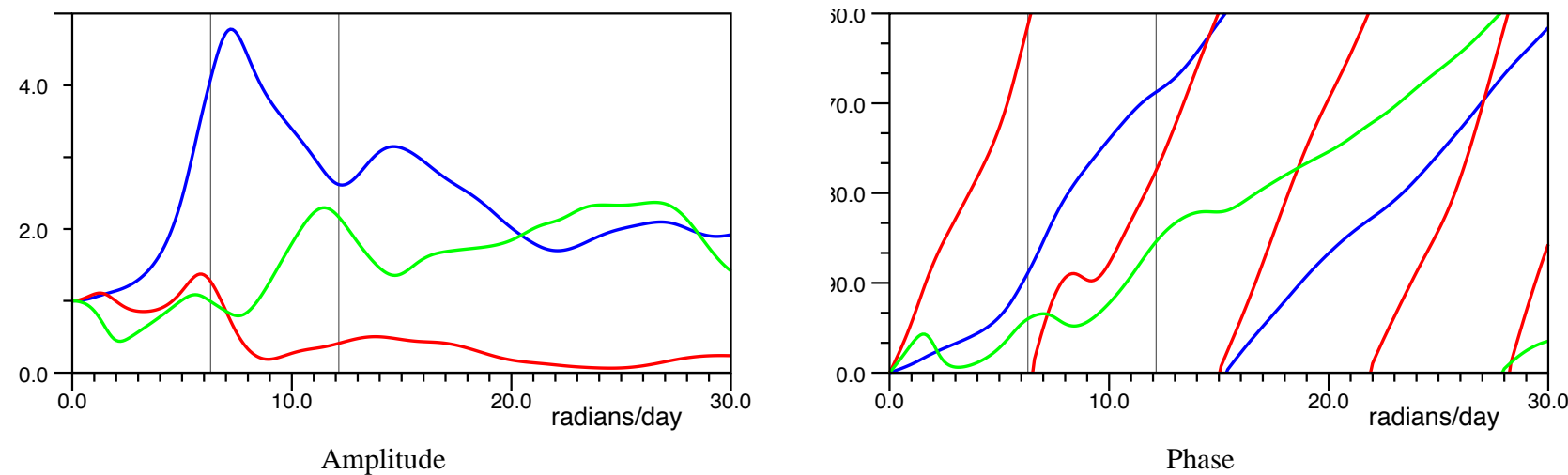

Fig. 5. Amplitude and Phase of the response functions between zero and 30 radians per day for Digoel River (blue), Karumba (red) and Yaboomba Island (green).

around 30 radians per day, but these are modulated by some structure which may be correlated with the Yaboomba Island response.

The Yaboomba Island station shows a third type of response. It starts low, and there is a small maximum near 5.5 radians per day, but after a second maximum near 11.5 radians per day, the amplitude stays high and is comparable to that at Digoel River.

The phase plots provide a different insight, in that each of the three curves tends to have a constant slope over all of the frequency range considered. There are changes in slope on the scale of the width of the peaks in the amplitude plot, but except for these features the slope remains remarkably constant.

One possible way to understand this behaviour is to consider the response of a simple travelling wave,

$\psi=A \exp (i k x-i \omega t)$

Assume that the wave speed is constant so that the $k$ equals $\omega / c$. Then, if this wave is "forced" so that it has amplitude one at the "boundary" where $x$ is zero, then at distance $x$, the response $R$ will be simply:

$$
\begin{aligned}
R(\omega) & =\exp (i k x), \\
& =\exp (i(x / c) \omega) .
\end{aligned}
$$

In this case the gradient of the phase equals $x / c$, the time taken for the wave to propagate from the boundary. Appendix $\mathrm{B}$ considers the case of a channel where the depth is not constant. This also shows that the phase is a linear function of $\omega$ with the slope again depending on the propagation time.

Applying this result to the phases of Fig. 5, it implies that on average incoming waves take about five hours to reach the Yaboomba Island from the open boundary, about ten hours to reach Digoel River and twenty-one hours to reach Karumba. The figures for Yaboomba Island and Karumba are in rough agreement for what might be expected from the phase lines of Figs. 2 and 3. These indicate that, above 10 radians per day, the tidal wave takes about four hours to reach Yaboomba Island and eighteen to twenty-four hours to reach Karumba.

Estimates for Digoel River are complicated by the standing waves affecting the semi-diurnal tide, but the diurnal tide indicates about four hours, which is much less than the ten hours estimated from Fig. 5. Thus, the simple idea of a progressive wave appears to be too simple, and an alternative picture needs to be developed.

\subsection{Resonance circles}

Webb (1973b) discusses the form of the ocean's response to tidal forcing and shows that it has the form:

$\psi(x, \omega)=\sum_{j} \psi_{j}(x) A_{j} /\left(\omega-\omega_{j}\right)$,

where $x$ denotes position, $\omega$ is the angular velocity, and the eigenvalue $\omega_{j}$ is the angular velocity of the $j$-th resonance. The eigenfunction $\psi_{j}(x)$ describes the spacial structure of the resonance, and $A_{j}$ depends on how the system is forced.

If the resonances are well separated, then near to each resonance the function at a fixed position has the form:

$\psi(\omega)=R_{j} /\left(\omega-\omega_{j}\right)+B(\omega)$.

where $B(\omega)$ is a smooth background, the contribution of distant resonances.

Let $\omega_{j}$ have real and imaginary parts $\omega_{j 0}$ and $\gamma_{j}$. In physically realistic systems with friction, $\gamma_{j}$ is negative. As $\omega$ moves along the real axis from minus to plus infinity, the resonance term increases from zero to a value of $i R_{j} / \gamma_{j}$ when $\omega$ equals $\omega_{j 0}$, and returns to zero at plus infinity. It is straightforward to show (Webb, 2011) that as this happens the real and imaginary components move anti-clockwise around a circle that starts at the origin and passes through $i R_{j} / \gamma_{j}$ at maximum distance from the origin.

Thus, it may be possible to identify resonances in observations or model results by plotting the real and imaginary components against each other and searching for clockwise circular features. However, if $B(\omega)$ is rapidly varying this 


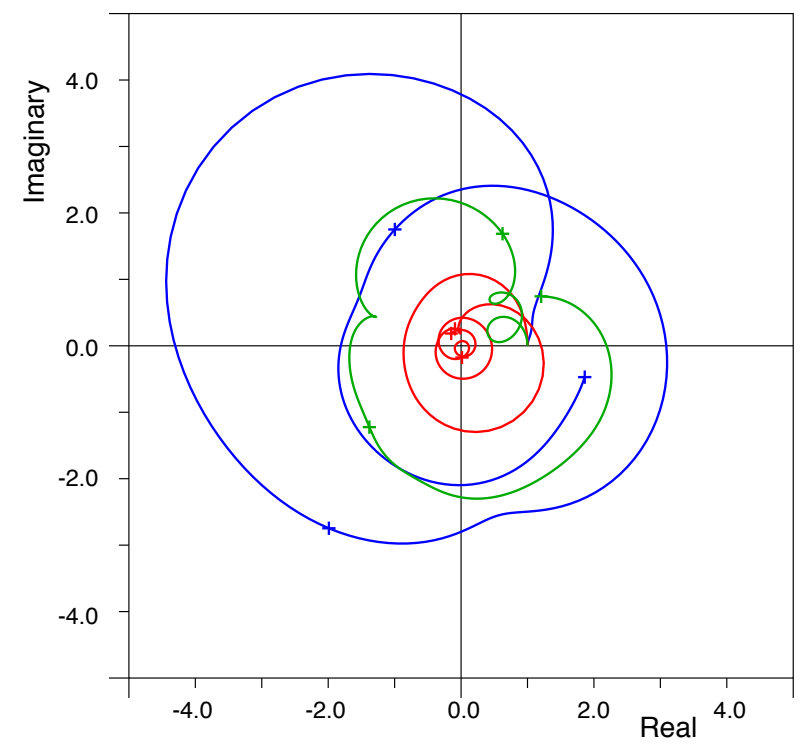

Fig. 6. Polar diagram showing the response functions between zero and 30 radians per day for Digoel River (blue), Karumba (red) and Yaboomba Island (green). The crosses are at intervals of 10 radians per day.

may be difficult. In particular, circles are also produced by delays, such as those produced by the propagation of progressive waves discussed in the last section, but in such cases the circles are centred on the origin. ${ }^{4}$

To see how well the approach works for the present results, the real and imaginary components are plotted against each other in Fig. 6. All three curves start at the point $(1.0+i 0.0)$ and circle around the origin in an anti-clockwise direction. However, although it is apparent that there are some underlying structures present in all three curves, only the Yaboomba Island curve shows anti-clockwise circles, and these are both very small and only occur at low angular velocities.

The main feature of the Karumba curve is that it circles the origin, initially at approximately unit distance, but finally it converges towards the origin. Thus, as discussed before it appears to be primarily a progressive wave which is damped at higher angular velocities.

The Digoel River curve also circles the origin but at a distance which is much greater than one, so this cannot be a simple delay. However, in the absence of an alternative explanation, it is possible that the resonances which produce the peaks of Fig. 5 are overlapping or combining in such a way as to produce an effective delay.

Figure 7 shows an attempt to remove the background by adding a linear correction to the phases, such that the total phase change over the whole band is zero. The transformation distorts the resonance circles, the distortion being pro-

\footnotetext{
${ }^{4}$ In terms of the resonance picture, a progressive wave is the cumulative result of many overlapping resonances.
}

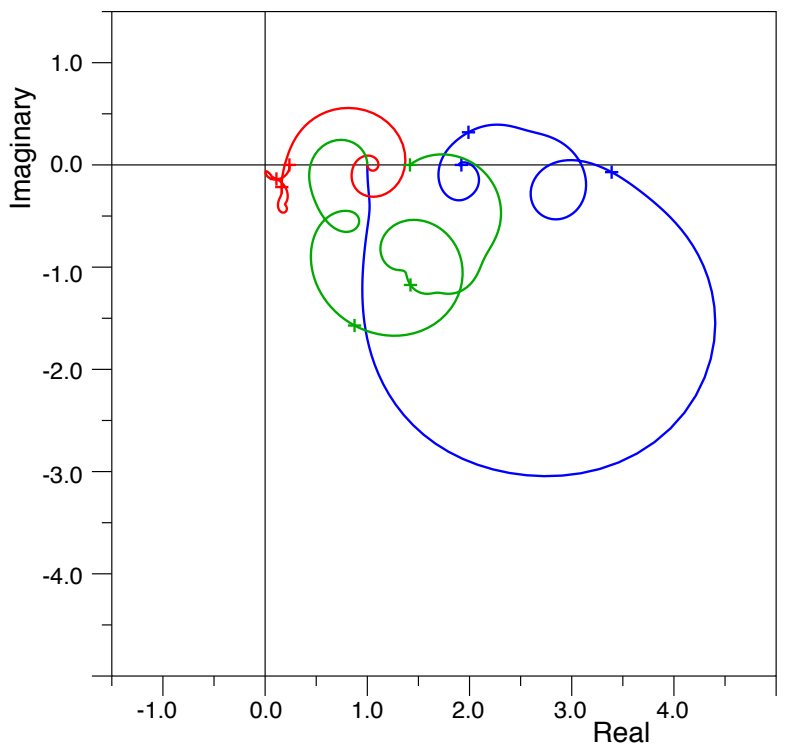

Fig. 7. Polar diagram showing the modified response functions (see text) between zero and 30 radians per day. Colours as in Fig. 6. The crosses are at intervals of 10 radians per day.

portional to the width of the resonance and the magnitude of the linear correction (greatest for Karumba), but the effect is small when the resonances are narrow.

Now all three stations show a series of loops with a positive (anti-clockwise) curvature, as expected from resonances. Karumba and Yaboomba Island both show some small kinks with negative curvature, but these will have been produced by the transformation.

\subsection{Analysis of real data}

The results of this section show that analysis based on results with real values of the angular velocity is difficult. Plots of just the real or imaginary components as a function of angular velocity are not very useful. Peaks in the amplitude plots start to indicate the presence of resonances, three or four at Digoel River and similar numbers at Yaboomba Island and Karumba but not all at the same angular velocities.

When additional confirmation is looked for in the phases, the main feature appears to be a steady change in phase indicating not resonances but delays in the system. The final method, plotting the real and imaginary components against each other, helps to confirm that resonances are being seen at Yaboomba Island, but at the other two stations, interpretation is again difficult.

It is possible that if all we had was data for real values of angular velocity, then more could be learnt by fitting the plotted data to a function similar to that of Eqs. (13) or (14). However, the present model can be run with complex values of angular velocity, and, as is shown in the next section, this allows a more complete exploration of the complex plane. 


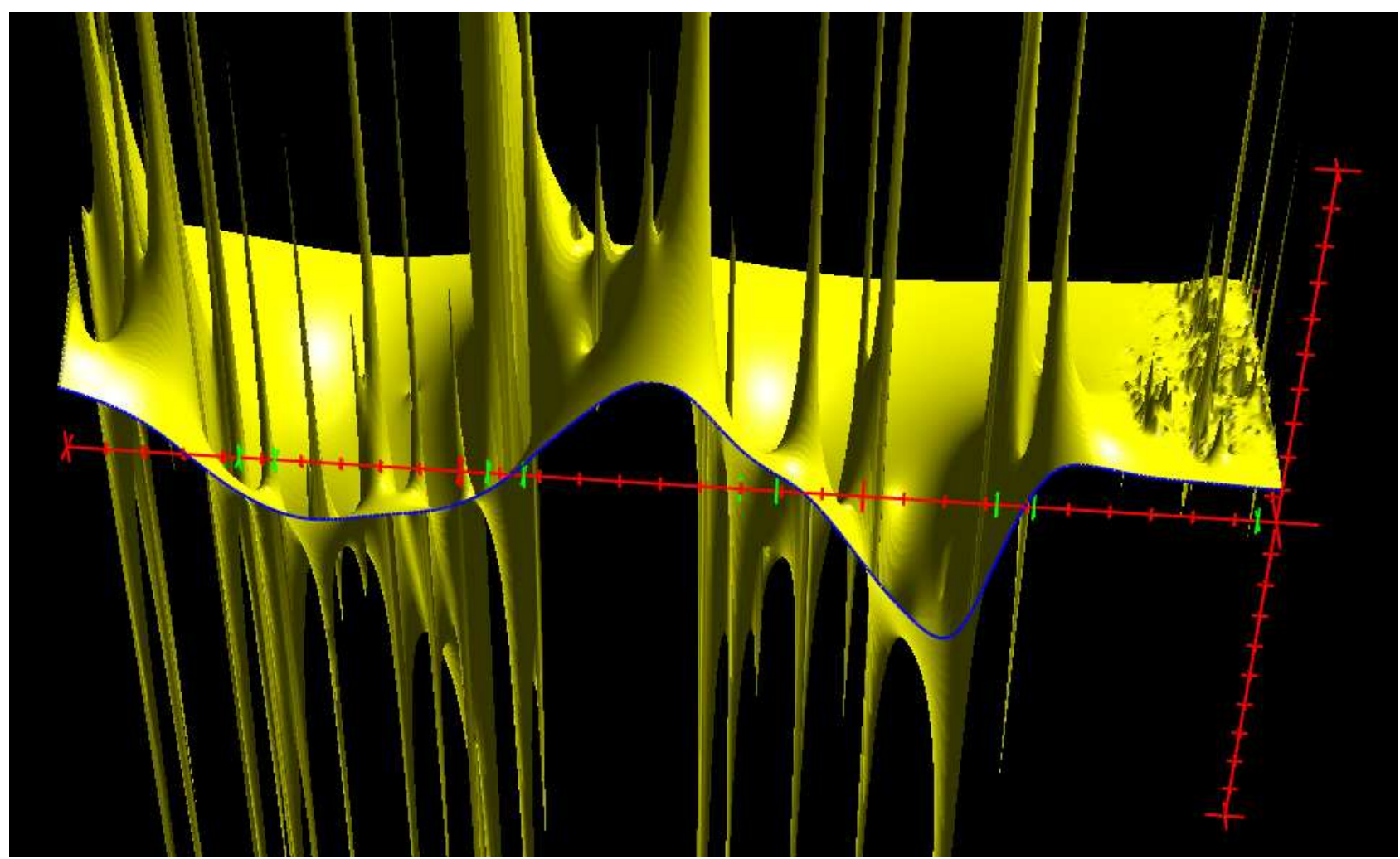

Fig. 8. Real part of the response function at Digoel River plotted as a function of complex angular velocity over the region enclosed by the origin and the points $(0-10 i),(30-10 i)$ and 30 radians per day. Axes in red with ticks every unit interval. The origin is on the right with the real axis extending to the left and the negative imaginary axis extending backwards. The green ticks indicate the long-period, diurnal, semi-diurnal and higher tidal bands. The blue line is the response at real angular velocities as plotted in Fig. 4 but with the direction of the real axis reversed.
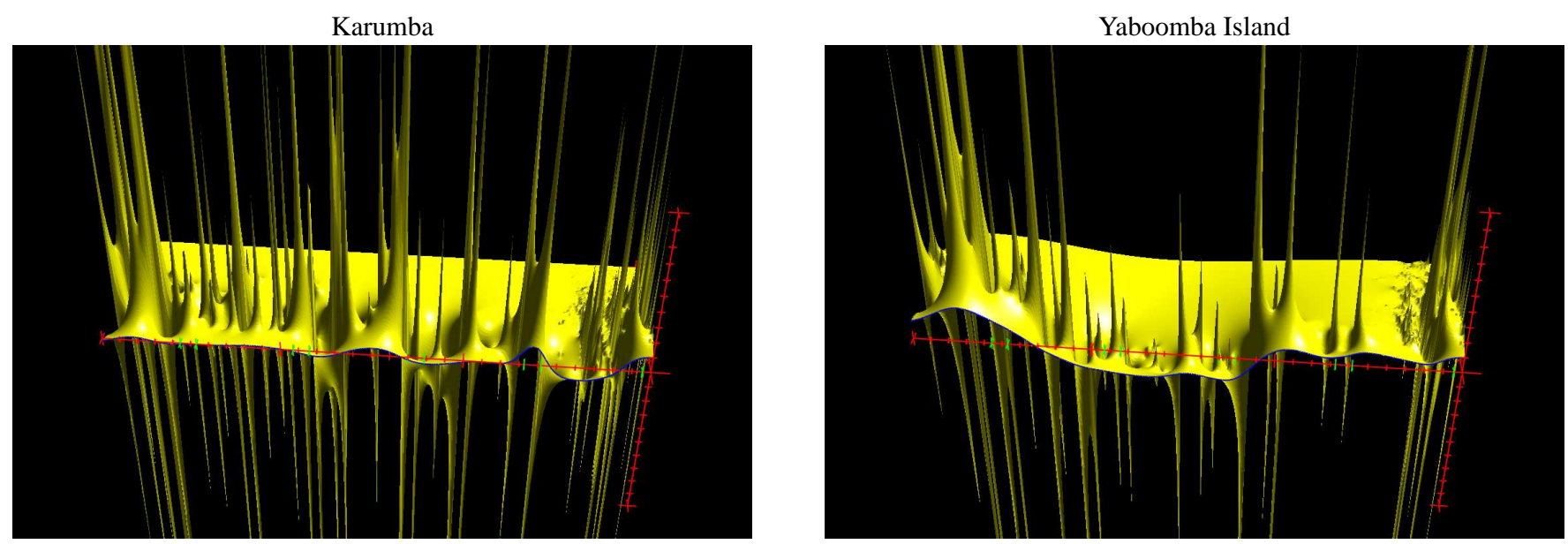

Fig. 9. Real part of the response functions at Karumba and Yaboomba Island. Details as in Fig. 8.

\section{The complex $\omega$ plane}

Figures 8 and 9 show the real component of the response at Digoel River, Karumba and Yaboomba Island plotted as a function of complex angular velocity. The data for the figures was created by running the model at intervals of 0.1 radians per day, in both the real and imaginary directions, over the rectangle whose corners are defined by the origin and the points $(0-10 i),(30-10 i)$ and 30 radians per day. The data 
was then plotted in a view which looks beyond the real axis in the negative imaginary direction.

The values on the real axis are the same as in Fig. 4, but the direction of increasing angular velocity is now towards the left. As discussed in Webb (1973b), the values in the negative real direction (not plotted) are the mirror image of those in the positive direction. This results from the fact that we are using a complex value response function to represent a system in which the input and output functions are both real.

The figures show series of mathematical poles at positions corresponding to the complex angular velocities of the resonances, the terms $\omega_{j}$ of Eq. (13). Although it is not always obvious, the poles are at the same positions in each of the three figures, and, if the resolution was high enough, the response functions would be seen to extend to plus and minus infinity in the neighbourhood of all of the poles.

The apparent width of each peak depends on the magnitude of its residue, i.e. the term $\psi_{j}(x) A_{j}$ in Eq. (13). Differences between the residues in the three figures is thus a measure of the changing importance of each resonance in different locations around the Gulf.

There should be no poles in the positive imaginary direction as these would correspond to modes which grow in time, and, as discussed by Webb (1973b) and in more detail by Nussenzveig (1972), such poles would also break causality. However, although the finite difference scheme used for the model is accurate to second order in the grid spacing, it is not strictly energy conserving, and so it is possible if the error terms are large that it could generate such modes. For the Gulf region this was found not to be the case.

The figures emphasise the large number of resonances found in even a small region of ocean and show that maxima on the real axis, i.e. in the real world, may not be due to an individual resonance but often result from the combined contributions of a number of resonances.

The figures also show that the resonances fall into two groups with a boundary near four radians per day. At low angular velocities there are a large number of tightly packed resonances, but they have little effect on the response at real values of angular velocity. In this group the residues are small, and there is a large range of decay rates, the imaginary components of angular velocity extending to minus ten and beyond.

Above four radians per day, the resonances are well separated and have much larger residues. At the lower (real) angular velocities, the imaginary components are one to two radians per day, and these increase to three to four radians per day for the resonances near thirty radians per day.

This second group of resonances is also the one which appears to have the most influence on the diurnal and semidiurnal tides. Table 1 contains a list of the resonances closest to the tidal bands plus resonances with low angular velocities which appear to have most effect on the low angular velocity response at Digoel River, Karumba and Yaboomba Island.

\subsection{Gravity and rossby waves}

Theoretical studies have shown that there are two main classes of long waves in the ocean. The first contains both the gravity waves, found at high angular velocities, and the equatorial and coastal Kelvin waves, which also extend to low angular velocities. In these waves the primary exchange is between the kinetic and potential energy of the wave. The second class of waves consists of the Rossby waves, which are found only at low angular velocities. In these waves the primary exchange is between the two horizontal components of velocity.

Theory also shows that the boundary between the gravity and Rossby waves occurs at an angular velocity of $f$, the Coriolis parameter,

$f=2 \Omega \sin (\theta)$,

where $\theta$ is latitude, and $\Omega$ is the angular velocity of the Earth. Waves with angular velocities near $f$ are likely to show a mixture of both properties (Longuet-Higgins, 1968; Longuet-Higgins and Pond, 1980).

The Gulf of Carpentaria and the Arafura Sea span latitudes between $5^{\circ} \mathrm{S}$ and $18^{\circ} \mathrm{S}$. This corresponds to values of the Coriolis parameter $f$, between 1.1 and 3.6 radians per day. Thus, the change in properties seen near 4 radians per day can be identified as being due to the change from the Rossby wave to the gravity wave parts of the spectrum.

If Eq. (1) is used to study the decay of a steady current, it is easy to show that the decay rate is proportional to $\kappa / h$, where $\kappa$ is the friction parameter and $h$ the depth. Webb (2011) investigated gravity wave resonances in a simple 1-D model with a constant depth continental shelf and showed that the resonance decay rates, the imaginary parts of the complex angular velocity, were given by the same equation.

In the present model, $\kappa$ has the value $0.1 \mathrm{~cm} \mathrm{~s}^{-2}$, and the depths range from over $100 \mathrm{~m}$ in parts of the Arafura sea and $70 \mathrm{~m}$ in the centre of the Gulf of Carpentaria to $20 \mathrm{~m}$ and less near to the coastline. Using the above equation, a depth of $100 \mathrm{~m}$ gives a decay rate of $0.86 \mathrm{day}^{-1}$ and this increases to $1.73 \mathrm{day}^{-1}$ for a depth of $50 \mathrm{~m}, 3.46 \mathrm{day}^{-1}$ for $25 \mathrm{~m}$ and 8.64 day $^{-1}$ for $10 \mathrm{~m}$. The gravity wave values of Table 1 are thus consistent with mean depths of between 25 and $50 \mathrm{~m}$, values which are not unreasonable.

Rossby waves on a shelf of constant depth are also expected to have decay rates of $\kappa / h$. Thus, the Rossby wave resonances of the current model with decay rates of 10 day $^{-1}$ must be in regions of the shelf where the depth is near $10 \mathrm{~m}$. There should also be gravity wave resonances with similar decay rates, but these presumably occur at much higher angular velocities than the range explored here. 

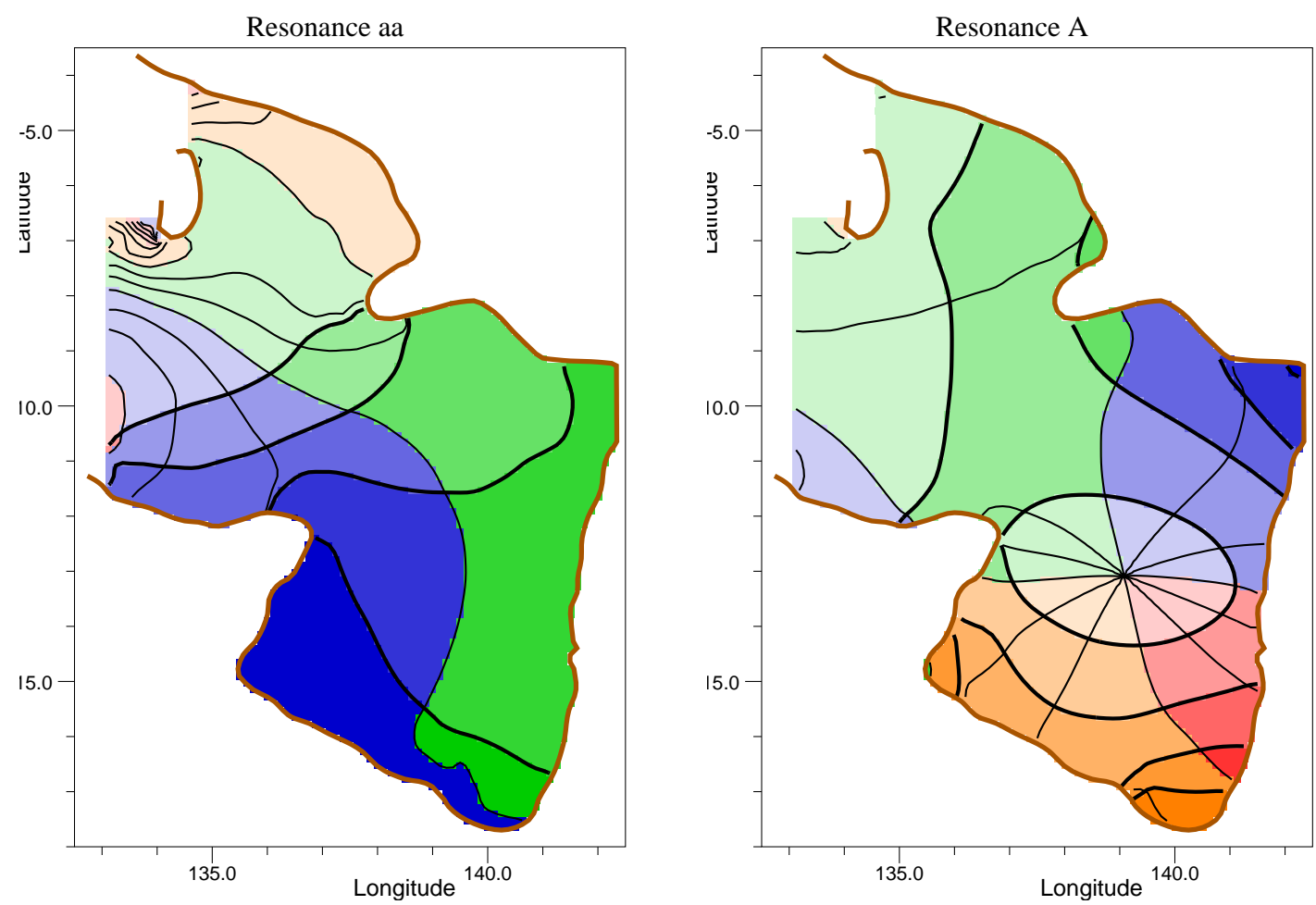

Fig. 10. Amplitude and phase contours for resonances aa and A (Table 1). The phase contours (light) are at intervals of 30 degrees, colour coded with red in the quadrant $-180^{\circ}$ to $-90^{\circ}$, brown $-90^{\circ}$ to $0^{\circ}$, green $0^{\circ}$ to $90^{\circ}$ and blue $90^{\circ}$ to $180^{\circ}$. Amplitude is is in the range 0 to 1 , with contours (heavy) at intervals of 0.2 and darker colours indicating greater amplitude. Amplitude is zero on the open (western) boundary and at the centre of amphidromes, and has the value 1 at points with maximum amplitude.

Table 1. Real and Imaginary components of angular velocity (in radians per day) for some of the key resonances, plus the names used to refer to them in the text.

\begin{tabular}{ccccccc}
\hline & \multicolumn{2}{c}{ Angular velocity } & & \multicolumn{2}{c}{ Angular velocity } \\
\cline { 2 - 3 } & Real & Imag. & & Real & Imag. \\
\hline aa & 1.4561 & -1.1419 & $\mathrm{~K}$ & 15.3736 & -1.6588 \\
bb & 3.1370 & -1.6373 & $\mathrm{~L}$ & 16.7251 & -2.0066 \\
$\mathrm{~A}$ & 5.8397 & -1.1277 & $\mathrm{M}$ & 16.9845 & -3.5121 \\
$\mathrm{~B}$ & 7.0215 & -1.3056 & $\mathrm{~N}$ & 17,4437 & -3.2135 \\
$\mathrm{C}$ & 7.8389 & -2.0418 & $\mathrm{O}$ & 18.4964 & -2.6164 \\
$\mathrm{D}$ & 9.5938 & -2.0780 & $\mathrm{P}$ & 19.1721 & -2.7670 \\
E & 10.2025 & -2.6180 & $\mathrm{Q}$ & 20.0978 & -3.4472 \\
F & 11.4272 & -1.7270 & $\mathrm{R}$ & 20.4820 & -1.8994 \\
G & 12.6226 & -2.4655 & $\mathrm{~S}$ & 20.6322 & -3.3178 \\
$\mathrm{H}$ & 13.3884 & -2.2500 & $\mathrm{~T}$ & 21.4129 & -1.9414 \\
$\mathrm{I}$ & 14.1413 & -2.2405 & $\mathrm{U}$ & 22.5463 & -2.6505 \\
J & 14.8486 & -2.5489 & & & \\
\hline
\end{tabular}

\section{The resonances}

The angular velocities of the resonances can be found by fitting Eq. (14) to one of the calculated response functions in the neighbourhood of each resonance. Use was made of the four nearest calculated values and these were fitted using a linear background,

$\psi(\omega)=R_{j} /\left(\omega-\omega_{j}\right)+A+B \omega$.

As discussed in Appendix A, this can be converted into a linear matrix equation and so solved for the unknowns including the resonance frequency $\omega_{j}$. The results for the main resonances using the data from Digoel River are given in Table 1. The results obtained from using any of the other stations are essentially the same.

Once the angular velocity of a resonance is known, the spatial structure, the eigenfunction of the matrix equation, can be calculated in a similar manner. This time, solutions to the model equations were obtained for each of the four angular velocities $\omega_{j} \pm \delta \omega$ and $\omega_{j} \pm \imath \delta \omega$, where $\delta \omega$ equalled 0.1 radian day $^{-1}$. Because the model uses Dirichlet boundary conditions, the eigenfunctions are, except for a normalising constant, independent of the details of the forcing used. Further information about the method, which makes use of the properties of Vandermonde matrices, is given in Appendix A. 

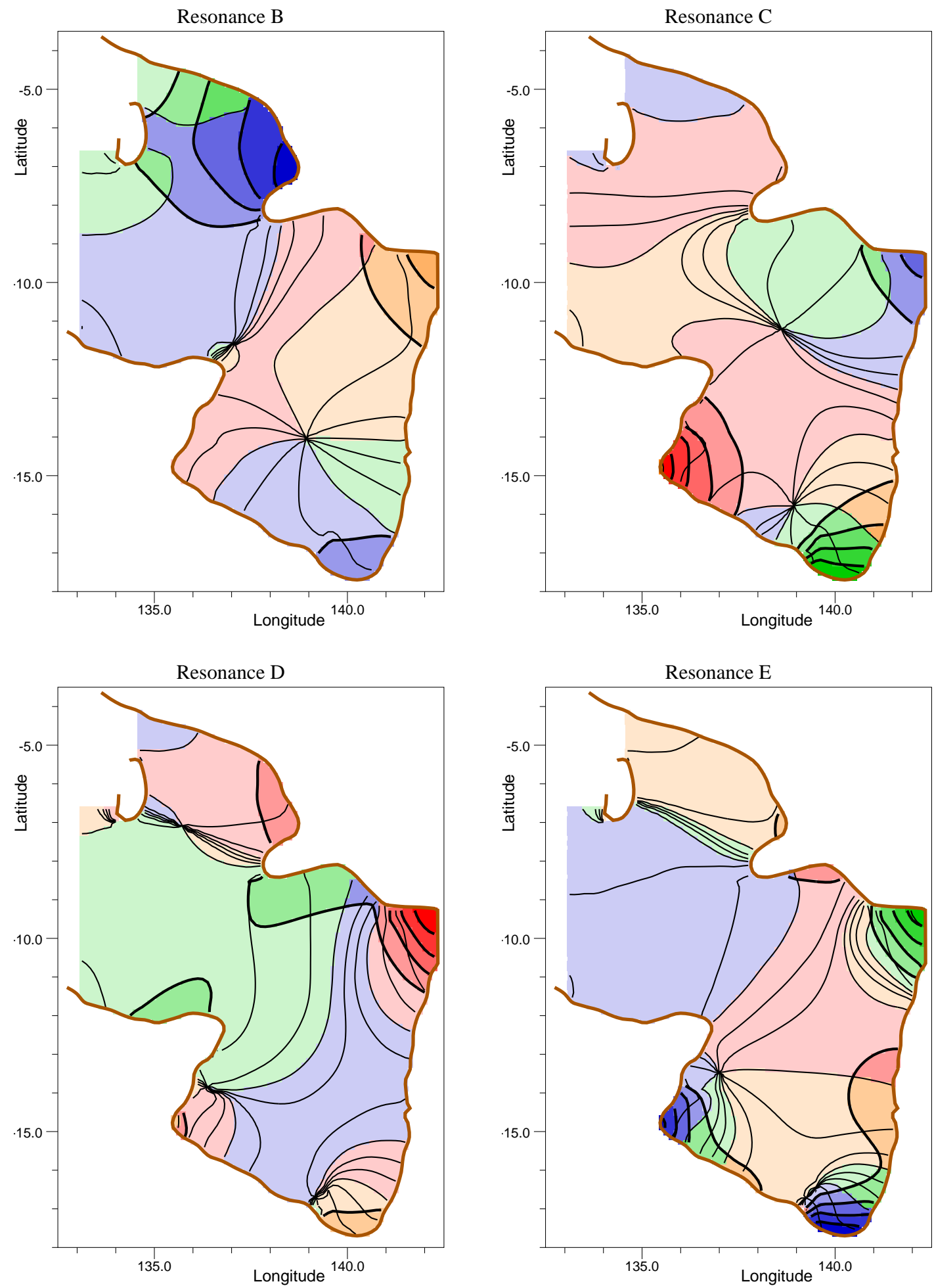

Fig. 11. Amplitude and phase contours for resonances B, C, D and E. Contours as in Fig. 10.

\subsection{Resonance waveforms}

Figures 10 to 13 show the amplitude and phase of some of the main resonances affecting the diurnal and semi-diurnal tides in the region. Mode aa is included because it is the fundamental quarter-wavelength mode, having a maximum at the southern limit of the Gulf of Carpentaria. However, it is not a classical quarter wavelength resonance because its angular 

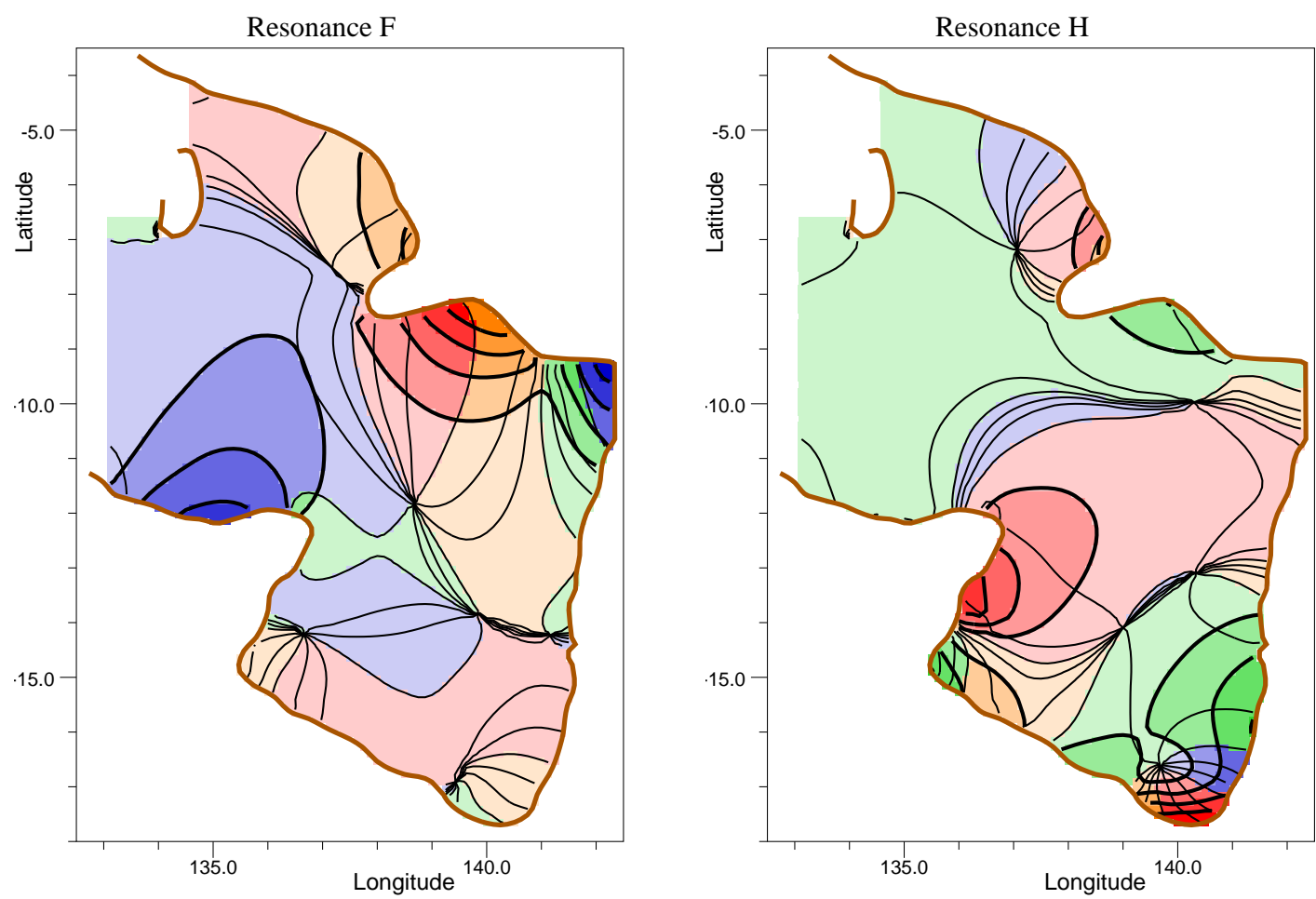

Fig. 12. Amplitude and phase contours for resonances F and H. Contours as in Fig. 10.

velocity is less than the Coriolis parameter everywhere except near the northern coastline of the Arafura Sea. As a result its properties are those of a Rossby wave with only a small fraction of its energy in the form of potential energy. Thus, even at low angular velocities it has only a limited effect on tidal height ${ }^{5}$.

The remaining resonances illustrated are all primarily gravity wave modes, and as such each of them might dominate the response with a suitable pattern of forcing. However, in the present case the forcing is limited to the shelf edge, and this appears to limit the importance of individual resonances and the way they interact.

At low angular velocities, resonances "A", "B" and "C" are well separated in angular velocity from other resonances and are relatively weakly damped. As a result one might expect them to dominate the response in the diurnal tide band, and, as discussed later, this is the case.

Figures 10 and 11 show that resonances " $A$ " and "C" are both primarily progressive waves trapped within the Gulf of Carpentaria. Their angular velocities appear to be determined by "A" fitting a single wavelength around a single amphidrome and "C" two wavelengths around a double amphidrome.

In contrast resonance "B" (Fig. 11) is primarily a quarter wavelength wave trapped between the shelf edge and the

\footnotetext{
${ }^{5}$ Grignon (2005) showed that the fundamental mode of the English Channel has similar properties
}

Digoel River. Some energy does propagate into and circulate around the Gulf of Carpentaria, but it appears to be the distance between the shelf edge and the Digoel River which determines the angular velocity of this mode. The angular velocities of resonances " $\mathrm{B}$ " and " $\mathrm{C}$ " are very close, so it is possible that this results in some mixing of the solutions.

At higher angular velocities the structure of the resonances becomes more complex, and it is more difficult to relate them to physical features. One exception is resonance "F", which appears to be primarily a standing wave trapped between the northern boundary of the Gulf of Carpentaria and the southern boundary of the Arafura Sea. Another is resonance "I", which is a three-quarters wave trapped between Digoel River and the shelf break. Note that this time there is very little coupling to the Gulf of Carpentaria.

The remaining resonances have a more irregular structure. Many of them, like resonance "E", have maxima in corners of the Gulf of Carpentaria, but the increasingly complex pattern of phases indicates that their main role is to provide the complete set of functions required to describe all possible wave patterns.

\subsection{Comparison with a simpler model}

Buchwald and Williams (1975) investigated the response of a simple rectangular gulf at the end of an infinite channel. They did not include a Coriolis term, but applying their model to the Gulf of Carpentaria, they found that the lowest 


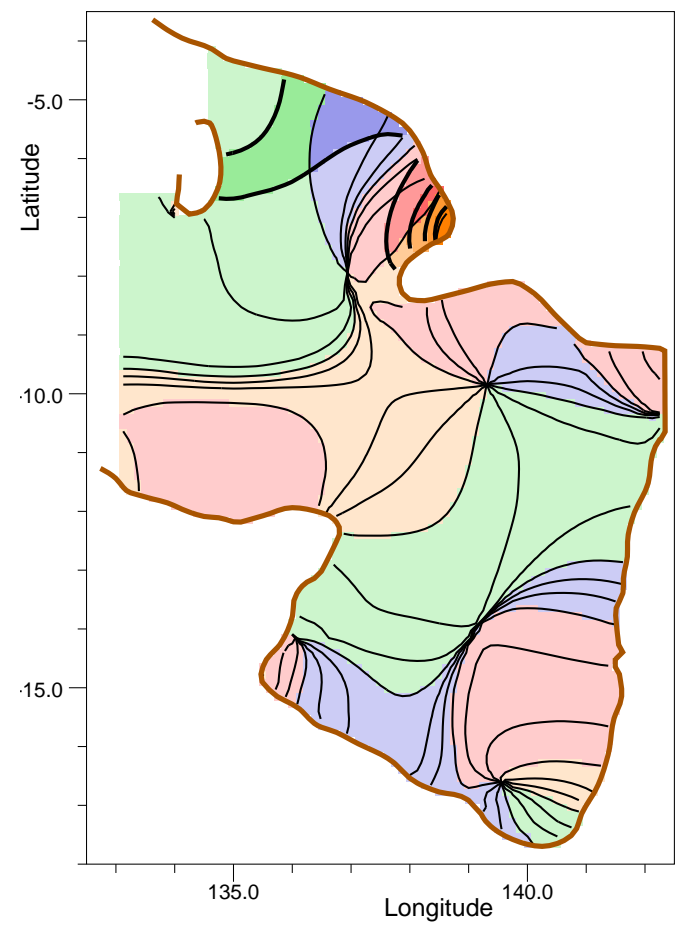

Fig. 13. Amplitude and phase contours for resonance I. Contours as in Fig. 10.

resonances had periods of $16.4,10.4$ and $7.9 \mathrm{~h}$, corresponding to $9.2,14.5$ and 19.1 radians per day, respectively. The results were given some support by Melville and Buchwald (1976), who analysed sea level records from the Gulf of Carpentaria.

One might have expected the Buchwald and Williams (1975) resonances to show similarities with those of the present study, which are most energetic in the Gulf of Carpentaria. The best examples are resonances $\mathrm{C}$ and $\mathrm{E}$, but these have angular velocities of 7.8 and 10.2 radians per day and do not correspond to any of the Buchwald and Williams (1975) values. This is most likely due to the lack of a Coriolis term in the earlier study.

\section{Resonances and real angular velocities}

The polar plots, discussed earlier, showed that at real values of the angular velocity the presence of resonances could be indicated by the presence of tight loops in the response function. Here, use is made of this property to identify the resonances responsible for the key features of the response function in the diurnal and semi-diurnal bands. Over such a band, it should be possible to expand the response function $R(x, \omega)$ at site $x$ as:

$R(x, \omega)=\sum_{j} R_{j}(x) /\left(\omega-\omega_{j}\right)+$ c.c. $+B(x, \omega)$,

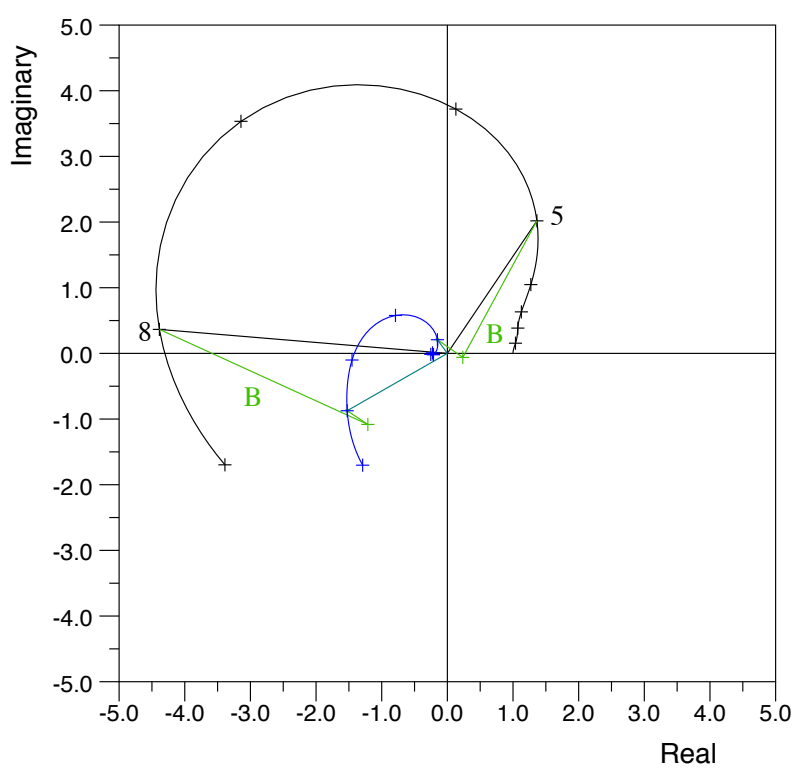

Fig. 14. Polar plot of response function (black) at Digoel River, for the range 0 to 9 radians per day. The black crosses are at intervals of one radian per day. The contribution of the resonance " $B$ ", and its conjugate pole (defined in the text), is plotted in green at 5 and 8 radians per day, the conjugate contribution being the smaller. The residual after subtracting the contribution of resonance " $\mathrm{B}$ " and its conjugate pole is plotted in blue, also for the range 0 to 9 radians per day.

where the sum $j$ is over a limited number of key resonances, and $B(x, \omega)$ is a smooth background. $R_{j}(x)$ and $w_{j}$ are the values calculated in the previous section. The term c.c. represents the contributions from the conjugate set of poles, with eigenvalues $-\omega_{j}^{*}$ and eigenfunctions $-R_{j}^{*}(x)$, needed for symmetry (Webb 1973b, Appendix I).

The choice of key resonances is to a certain extent subjective. In the examples given here, it was done by finding a set of resonances which, over the band of real angular velocities considered, reproduced the main features of the response function and left a residual which was small and smooth. As a final check the other nearby resonances were each added in turn but then left out if they did not significantly smooth or reduce the residual.

The results are illustrated using the polar form of the response function figures. The full response function and the background term are plotted for the whole band, and the individual contributions from the selected resonances and their complex conjugates are plotted at two points within each band. Because of the difference in angular velocity, the complex conjugate contributions tend to be small except near zero angular velocity. 


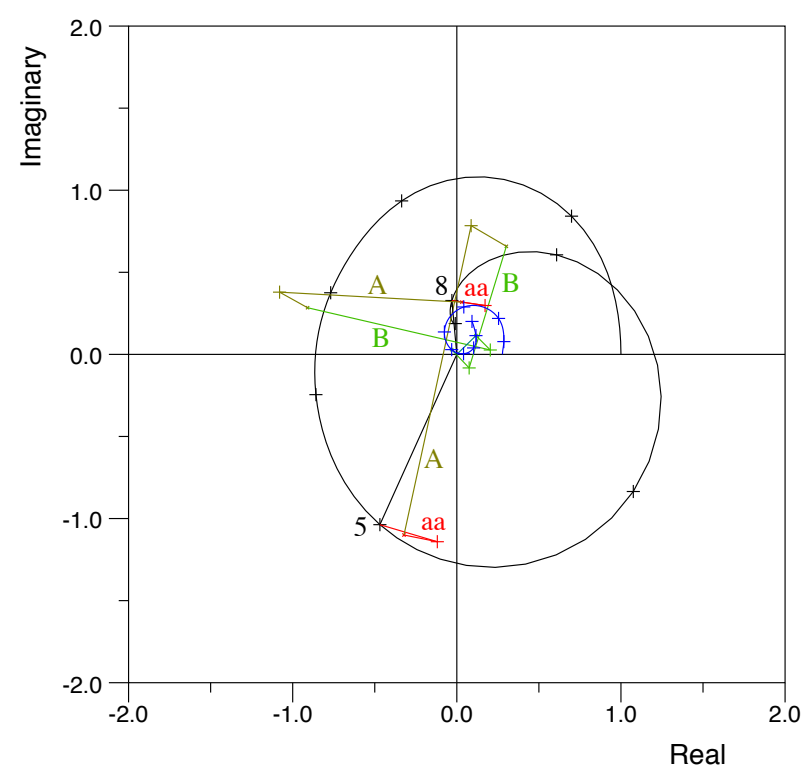

Fig. 15. Polar plot of response function (black) at Karumba, for the range 0 to 9 radians per day. The black crosses are at intervals of one radian per day. The contribution of the resonances "aa" (red), "A" (yellow) and "B" (green), and their conjugate poles, are plotted at 5 and 8 radians per day, the conjugate contribution being the smaller. The residual, after subtracting the contributions of these resonances and their conjugates, is plotted in blue, also for the range 0 to 9 radians per day.

Two bands of angular velocities are considered. The first extends from zero to nine radians per day. This covers the diurnal tides and also shows the behaviour at very low values of angular velocity. The second band extends from ten to fifteen radians per day and covers the semi-diurnal tides.

\subsection{The diurnal band}

The result for Digoel River, for the band 0 to 9 radians per day is shown in Fig. 14. The large amplitude loop in the response function can all be explained by the changing amplitude and phase of the contribution of resonance "B", a quarter wavelength resonance. The background term is seen to grow with angular velocity, reaching an amplitude of two by 8 radians per day, but it still remains much smaller than the single resonance contribution.

The corresponding result for Karumba is shown in Fig. 15. At low angular velocities it was found necessary to include resonance "aa" in order to reduce the background at near zero angular velocity. For the 5 to 8 radians per day region, which includes the diurnal tides, it was found necessary to include both "A" and "B" resonances. These both have large amplitudes and phase changes of around $90^{\circ}$.

More importantly they have approximately opposite phase. Near 5 radians per day, the contribution from resonance "A" is large and "B" only reduces it slightly. How-

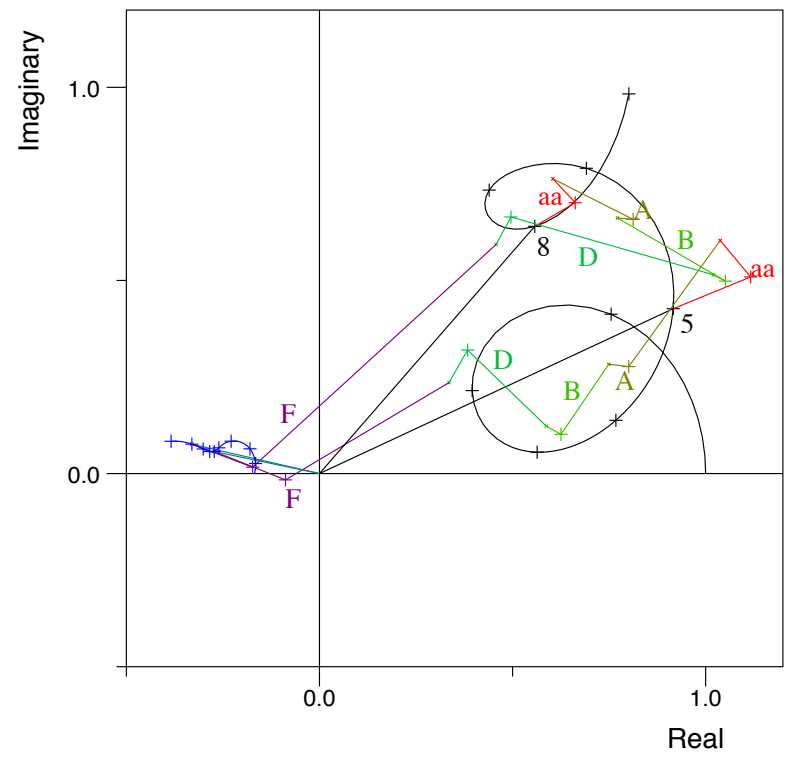

Fig. 16. Polar plot of response function (black) at Yaboomba Island, for the range 0 to 9 radians per day. The black crosses are at intervals of one radian per day. The contribution of the resonances “aa" (red), "A" (yellow), "B" (light green), "D” (dark green), and "F" (purple), and their conjugate poles, are plotted at 5 and 8 radians per day, the conjugate contribution being the smaller. The residual, after subtracting the contributions of these resonances and their conjugates, is plotted in blue, also for the range 0 to 9 radians per day.

ever, by 8 radians per day they are effectively cancelling each other out, and this results in a much lower amplitude response function. Figures 2 and 3 show that the semi-diurnal tides at Karumba are low compared with the diurnal tides, and this might have been thought to be a result of an increase in the effect of friction at higher angular velocities. However, in the present case both modes have similar decay times, and so the reduction in amplitude is purely an effect of interference between the two modes.

A further complication is found is found in the results for Yaboomba Island (Fig. 16). The first loop is found to be primarily the effect of the Rossby wave resonance "aa". Resonances " $\mathrm{A}$ " and "B" are also significant. They again show an approximately $90^{\circ}$ phase change between 5 and 8 radians per day, but here they are acting in phase and as a result produce the increase in amplitude near 5 radians per day. Two more resonances, "D" and "F", are also significant, and as the angular velocity increases they grow even larger. From Figs. 11 and 12, they both involve a standing wave between the southern boundary of the Arafura Sea and the north of the Gulf of Carpentaria. Together they both contribute towards completing the second loop of the response function and the further increase in its amplitude past 8 radians per day. 


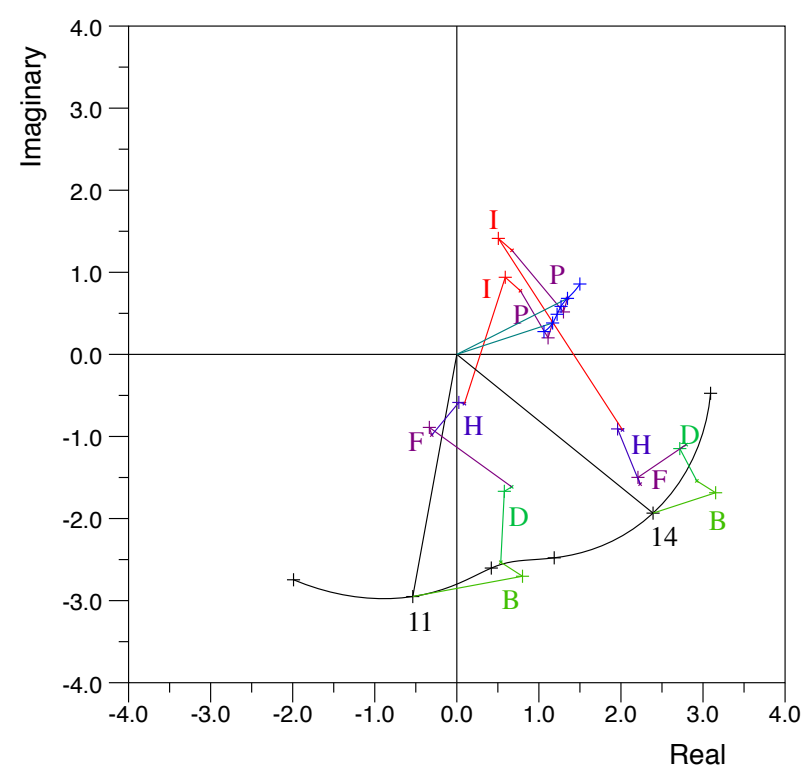

Fig. 17. Polar plot of response function (black) at Digoel River, for the range 10 to 15 radians per day. The black crosses are at intervals of one radian per day. The contribution of the resonances "B", "D", "F", "H", "I", and "P", and their conjugate poles, are plotted at 11 and 14 radians per day, the conjugate contribution being the smaller. The residual, after subtracting the contributions of these resonances and their conjugates, is plotted in blue, also for the range 10 to 15 radians per day.

\subsection{The semi-diurnal band}

Figure 17 shows the response function at Digoel River in a range including the semi-diurnal tides. Resonance " $\mathrm{B}$ " is still significant, but it is declining in importance and a large number of other resonances are now involved. These include "D", "F", "H" and "P", all of which show noticeable phase changes between 11 and 14 radians per day, but the largest contribution is now from "I", the three-quarter wave resonance trapped between the Digoel River and the shelf edge.

At Karumba, Fig. 18, all of the resonances in the range " $D$ " to "K" now make significant contributions. The largest amplitude term is that of " $\mathrm{H}$ ", a rather complex resonance confined to the Gulf, but its effect is to a large extent cancelled out by other resonances. However, its increased amplitude does seem to be responsible for the second partial loop seen around 14 radians per day.

Cancellation is also seen within the group of resonances "E", "F", and "G". Resonances "A", "B", and "C" (which for clarity are not plotted) cancel each other in a similar manner.

In contrast to Digoel River and Karumba, the number of significant resonances contributing to the response function at Yaboomba Island has dropped (see Fig. 19). "F" is the largest resonance, responsible for the main loop, and only two more, "D" and "K", are required to smooth the back-

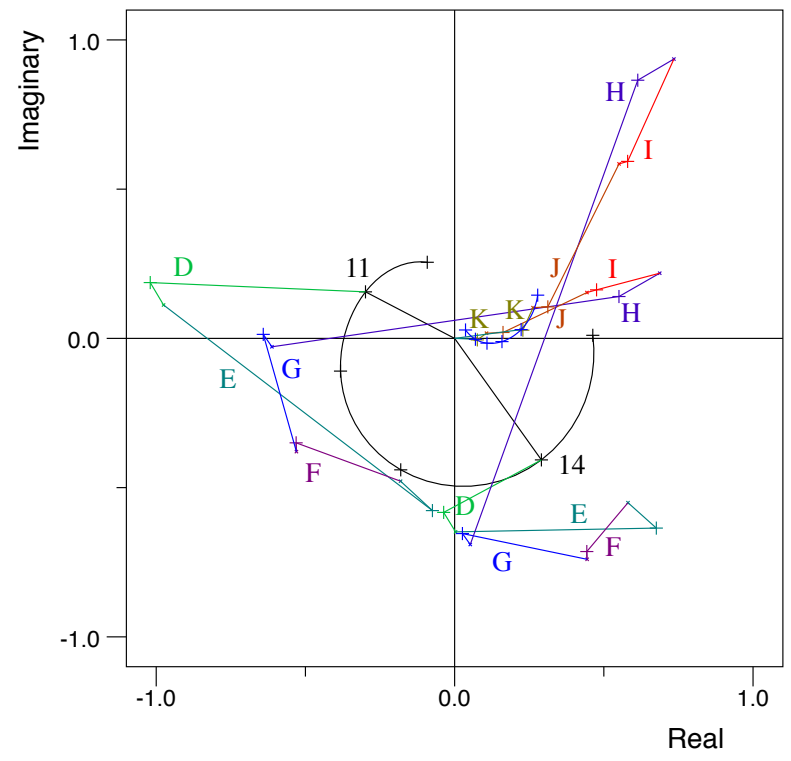

Fig. 18. Polar plot of response function (black) at Karumba, for the range 10 to 15 radians per day. The black crosses are at intervals of one radian per day. The contribution of the resonances " $D$ " to " $K$ ", and their conjugate poles, are plotted at 11 and 14 radians per day, the conjugate contribution being the smaller. The residual, after subtracting the contributions of these resonances and their conjugates, is plotted in blue, also for the range 10 to 15 radians per day.

ground. The result confirms that the unusual standing wave between the southern boundary of the Arafura Sea and the northern boundary of the Gulf of Carpentaria is a key feature of the region.

\section{Conclusions}

The results demonstrate the advantages of linearised tidal models which can work with complex values of angular velocity. In reality non-linear processes occur in the ocean, but although locally they may be important, the large scale response of the ocean to tidal forcing is predominantly linear. Of course the linearisation process needs to be done carefully, and if possible validated, but it does provide a different viewpoint for the study of the ocean.

Tidal models which use a timestepping scheme are limited to real values of angular velocity. When the present model was limited in the same way, the results were unsatisfactory. Plots of the real and imaginary components of the response function and its amplitude showed peaks that one might be tempted to attribute to resonances. However, these were not simple ones that one may expect from a single resonance and the peaks occurred at different angular velocities in different parts of the study region. Interpretation was further complicated by the phase plots which indicated that the response may be largely in the form of a simple progressive 


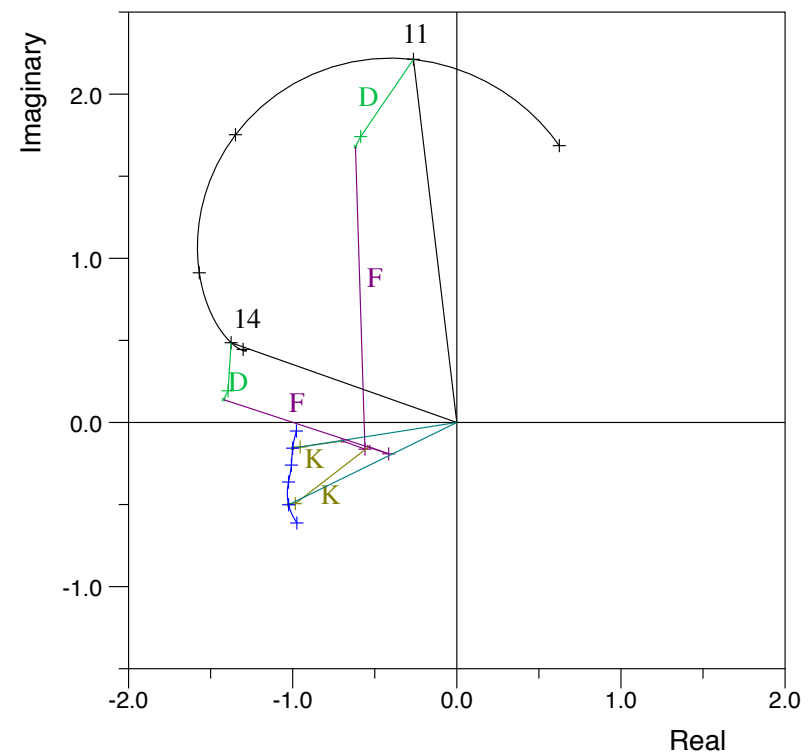

Fig. 19. Polar plot of response function (black) at Yaboomba Island, for the range 10 to 15 radians per day. The black crosses are at intervals of one radian per day. The contribution of the resonances "D", "F" and "K", and their conjugate poles, are plotted at 11 and 14 radians per day, the conjugate contribution being the smaller. The residual, after subtracting the contributions of these resonances and their conjugates, is plotted in blue, also for the range 10 to 15 radians per day.

wave only slightly modified by other processes. The polar diagrams were more informative as they showed a number of tight loops with positive curvature. Theory predicts that isolated resonances will generate such loops.

In principle it should be possible go further by analytically continuing the response function from the real axis to complex values of angular velocity. However, such a process can be affected by numerical noise and so is likely to be practical only for well separated resonances close to the real axis.

When the restriction was lifted and the model run for complex values of angular velocity, the results were much more informative, the three dimensional figures showing a complex pattern of resonances. This included a series of poles with large residues extending from six radians per day to higher angular velocities. These were identified as the gravity wave resonances of the region. There was also a field of smaller poles with small real angular velocities but with a large range of imaginary angular velocities. These were identified as the Rossby wave modes.

An investigation of the eigenfunctions showed that some of the gravity modes, especially those with low (real) angular velocities, could be identified as fundamental $1 / 4$ or $3 / 4$ wavelength modes fitting between the shelf edge and the coast or modes associated with reflection between opposing boundaries. Others were much more complex, whose main role may be to contribute to the complete set of modes needed to describe all possible waveforms within the system.

The Rossby wave modes were not investigated in the same way, but one of them was found to be a $1 / 4$ wavelength wave trapped between the shelf edge and the southern boundary of the Gulf of Carpentaria. With no Coriolis term this would be the fundamental quarter wavelength mode of the system and so would have a significant impact on the sea level response. However, here it is at such a low angular velocity that it is primarily a Rossby wave and, except near Yaboomba Island where the other terms are small, it has limited impact on the sea level response.

These results were then used to reinterpret the response functions calculated on the real axis. This showed that there is no band of real angular velocities where the response can be represented by the contribution from a single resonance. However, close to the diurnal tidal band there are only a few resonances, and, although a background term is also required, these were found to dominate the response within the band. There are more resonances close to the semi-diurnal band, and the behaviour there was found to be more complicated.

The results also show that in both bands there are regions of the continental shelf where the response is strongly influenced by resonances trapped between the shelf edge and the coast or between two opposing coastlines. Elsewhere the resonances often appear to produce cooperative effects, a group of resonances forming a single "resonant like" loop, cancelling each others contributions or producing an approximation to a simple progressive wave.

This apparent cooperation may simply reflect the fact that in such regions the separation between resonances is less than their distance from the real axis. Alternatively, it may be an indication that, where it occurs, the physical process involved is one for which a simple resonance interpretation is the wrong one. This should occur, for example, in the case of a simple progressive wave. The resonance picture may also be inappropriate for a slowly narrowing channel many wavelengths long, for which a Wentzel-Kramers-Brillouin (WKB) type approximation may be more relevant. However, the fact that the $1 / 4,3 / 4$ and other simple resonances stand out so clearly implies that such resonances are still likely to be important in other regions of the world's ocean.

Finally, although the study has concentrated on the properties of the Gulf region, the main implication of the present study is a more general one. It is that because the tides are approximately linear and because friction is involved, there is much to be gained from the use of linearised models to study the ocean's response to tidal forcing, especially when combined with the well known properties of linear operators. The approach generates accurate resonance eigenvalues and eigenfunctions, and, as the results presented here show, it can also provide new insights into the way the resonances interact and the physical processes that they represent. 


\section{Appendix A}

\section{Resonance and background}

The problem is to fit a series of response function values $R\left(\omega_{i}\right)$ for different values of $\omega_{i}$ by a single resonance and a linear background,

$R\left(\omega_{i}\right)=R_{j} /\left(\omega_{i}-\omega_{j}\right)+P+Q \omega_{i}$.

$P$ and $Q$ are complex constants, $\omega_{j}$ is the complex angular velocity of the resonance, and $R_{j}$ its residue. This is a specific form of the more general problem of fitting a set of values $y_{i}$ and $x_{i}$ to an equation of the form:

$y=A /(x-B)+\sum_{n=0}^{N} C_{n} x^{n}$.

Multiplying by $(x-B)$,

$$
\begin{aligned}
y(x-B)= & A+\sum_{n=0}^{N}\left(C_{n} x^{n+1}-C_{n} B x^{n}\right), \\
y x= & A+B y-C_{0} B \\
& +\sum_{n=1}^{N}\left(C_{n-1}-C_{n} B\right) x^{n}+C_{N} x^{N+1}, \\
y x= & \left(A-C_{0} B\right)+B y \\
& +\sum_{n=1}^{N}\left(C_{n-1}-C_{n} B\right) x^{n}+C_{N} x^{N+1} .
\end{aligned}
$$

This can be written in the form:

$y x=D_{0} y+\sum_{n=1}^{N+2} D_{n} x^{n-1}$.

This, like Eq. (A2), has $\mathrm{N}+3$ unknowns. If a similar number of pairs of values $x$ and $y$ are available, it gives a matrix equation which can be solved using standard methods for the unknowns $\boldsymbol{D}$. Then

$$
\begin{aligned}
B & =D_{0}, \\
C_{N} & =D_{N+2}, \\
C_{n-1} & =D_{n+1}+C_{n} B \quad \text { for } \quad n=1 \ldots N, \\
A & =D_{1}+C_{0} B .
\end{aligned}
$$

If in Eq. (A1) the angular velocity of the resonance $\omega_{j}$ is known, then Eq. (A2) can be rewritten in the form:

$y=A / x^{\prime}+\sum_{n=0}^{N} C_{n}^{\prime} x^{\prime n}$,

where $x^{\prime}$ now equals $\left(w_{i}-w_{j}\right)$. Dropping the primes, the equivalent of Eq. (A4) is:

$y x=\sum_{n=0}^{N+1} D_{n} x^{n}$.
In matrix form, the set of equations become:

$\mathbf{M} \boldsymbol{D}=\boldsymbol{F}$,

where $\mathbf{M}$ is a matrix, and $\boldsymbol{D}$ and $\boldsymbol{F}$ are vectors,

$M_{m, n}=x_{m}^{n}$,

$F_{m}=y_{m} x_{m}$.

$\mathbf{M}$ is a Vandermonde matrix. Turner (1966) shows that its inverse, $\mathbf{M}^{-1}$, contains terms which are simple functions of the variables $x_{m}$. The solution is then given by

$\boldsymbol{D}=\mathbf{M}^{-1} \boldsymbol{F}$.

The residue of the pole in Eq. (A6) is given by $D_{0}$. In the case of four unknowns, if the $x_{i}$ s have the values $\pm \delta$ and $\pm i \delta$, where $\delta$ is non-zero, then the residue is just the mean value of the elements of $\boldsymbol{F}$.

\section{Appendix B}

\section{Shallow water solution in a channel}

Consider a channel of constant width where the depth $H(x)$ varies with distance $x$ along the channel. The shallow water equations are then:

$\mathrm{d} u / \mathrm{d} t=-g \mathrm{~d} h / \mathrm{d} x$,

$\mathrm{d} h / \mathrm{d} t=-d(H u) / \mathrm{d} x$,

where $h$ is sea level, $u$ is velocity, $t$ is time, and $g$ is gravity. If the waves have constant angular velocity $\omega$, then after eliminating $u$ and dropping the time dependent term, $\exp (-i \omega t)$,

$h^{\prime \prime}+\left(H^{\prime} / H\right) h^{\prime}+\left(\omega^{2} /(g H)\right) h=0$,

where $h^{\prime}$ and $h^{\prime \prime}$ represent $\mathrm{d} h / \mathrm{d} x$ and $\mathrm{d} h^{2} / \mathrm{d} x^{2}$. Let $k$ equal $\left(\omega^{2} /(g H)\right)^{1 / 2}$. It has the properties of a local wavenumber. Then

$h^{\prime \prime}-\left(2 k^{\prime} / k\right) h^{\prime}+k^{2} h=0$.

If the depth changes slowly over each wavelength, a method similar to the WKB approximation can be used (Gill, 1982). Let

$h(x)=A \exp (i \phi(x))$.

Substitute in Eq. (B3), equate the real and imaginary components and simplify:

$$
\begin{aligned}
\phi^{2} & =k^{2}+\left[A^{\prime \prime} / A-2\left(k^{\prime} / k\right)\left(A^{\prime} / A\right)\right], \\
2 A^{\prime} k^{\prime} & =A\left(2\left(k^{\prime} / k\right) \phi^{\prime}-\phi^{\prime \prime}\right) .
\end{aligned}
$$

If the amplitude and the depth change slowly so that the term in square brackets is small,

$\phi^{\prime} \approx \pm k$,

$A \approx C k^{1 / 2}$, 
where $C$ is a constant. Thus

$$
\begin{aligned}
h(x) & \approx C k(x)^{1 / 2} \exp \left( \pm i \int k(x) \mathrm{d} x\right), \\
& \approx C k(x)^{1 / 2} \exp \left( \pm i \omega \int(g H(x))^{-1 / 2} \mathrm{~d} x\right) .
\end{aligned}
$$

At a fixed point along the channel, the phase of the solution is linearly proportional to the angular velocity of the wave.

Acknowledgements. I wish to thank the editors of the CSIRO journal "Marine and Freshwater Research" for permission to reuse figures from Webb (1981).

Edited by: J. M. Huthnance

\section{References}

Anon: Admiralty Tide Tables, Hydrographer of the Navy, London, 1971.

Arbic, B. K. and Garrett, C.: A coupled oscillator model of shelf and ocean tides, Cont. Shelf Res., 20, 564-574, doi:10.1016/j.csr.2009.07.008, 2010.

Arbic, B. K., St-Laurent, P., Sutherland, G., and Garrett, C.: On the resonance and influence of the tides in Ungava Bay and Hudson Strait, Geophys. Res. Lett, 34, L17606, doi:10.1029/2007GL030845, 2007.

Arbic, B. K., Karsten, R. H., and Garrett, C.: On Tidal Resonance in the Global Ocean and the Back-Effect of Coastal Tides upon Open-Ocean Tides, Atmos.-Ocean, 47, 239-266, 2009.

Buchwald, V. T. and Williams, N. V.: Rectangular resonators on infinite and semi-infinite channels, J. Fluid Mech., 67, 497-511, 1975.

Duff, G.: Tidal resonance and tidal barriers in the Bay of Fundy system, J. Fish. Res. Board Can., 27, 1701-1728, 1970.

Easton, A. K.: The tides of the continent of Australia, Report 37, Horace Lamb Centre for Oceanographic Research, Flinders University of South Australia, 1988.

Egbert, G. and Ray, R.: Semi-diurnal and diurnal tidal dissipation from TOPEX/Poseidon altimeter data, Geophys. Res. Lett., 30, 1907, doi:10.1029/2003GL017676, 2003.

Fong, S. and Heaps, N.: Note on the quarter-wave tidal resonance in the Bristol Channnel, Institute of Oceanographic Sciences, Report No., 63, 1978.

Garrett, C. and Munk, W.: The age of the Tide and the 'Q' of the Ocean, Deep-Sea Res., 18, 493-504, 1971.

Gill, A.: Atmosphere-Ocean Dynamics, Academic Press, New York \& London, 662 pp, 1982.

Green, J. A. M.: Ocean tides and resonances, Ocean Dynam., 60, 1243-1253, 2010.
Griffiths, S. D. and Peltier, W. R.: Megatides in the Arctic Ocean under glacial conditions, Geophys. Res. Lett., L08605, doi:10.1029/2008GL033263, 2008.

Griffiths, S. D. and Peltier, W. R.: Modeling of Polar ocean Tides at the Last Glacial Maximum: Amplification, Sensitivity, and Climatological Implications, J. Climate, 22, 2905-2924, 2009.

Grignon, L.: Tidal resonances on the North-West European Shelf, Master's thesis, University of Southampton, School of Ocean and Earth Science, 2005.

Longuet-Higgins, M.: The Eigenfunctions of Laplace's Tidal Equations over a Sphere, Philos. Trans. Roy. Soc., A262, 511-607, 1968.

Longuet-Higgins, M. and Pond, G. S.: The Free Oscillations of Fluid on a Hemisphere Bounded by Meridians of Longitude, Philos. Trans. Roy. Soc., A266, 193-223, 1980.

Melville, W. and Buchwald, V.: Oscillations of the Gulf of Carpentaria, J. Phys. Oceanogr., 6, 394-398, 1976.

Miller, G. R.: The flux of tidal energy out of the deep ocean, J. Geophys. Res., 71, 2485-2489, 1966.

Müller, M.: Synthesis of forced oscillations, Part 1: Tidal dynamics and the influence of the loading and self-attraction effect, Geophys. Res. Lett., 34, L05606, doi:10.1029/2006GL028870, 2007.

Nussenzveig, H. M.: Causality and Dispersion Relations, Academic Press, 1972.

Turner, L. R.: Inverse of the Vandermonde Matrix with Applications, NASA Technical Note D-3547, US National Aeronautics and Space Administration, 1966.

Webb, D.: On the Age of the Semi-diurnal Tide, Deep-Sea Res., 20 847-852, 1973a.

Webb, D. J.: Green's Function and Tidal Prediction, Rev. Geophys Space Phys., 12, 103-116, 1973b.

Webb, D. J.: A Model of Continental Shelf Resonances, Deep-Sea Res., 23, 1-15, 1976.

Webb, D. J.: Numerical Model of the Tides in the Gulf of Carpentaria and Arafura Sea, Austr. J. Mar. Freshw. Res., 32, 31-44, 1981.

Webb, D. J.: Tides and Tidal Energy, Contemp. Phys., 23, 419-442, 1982.

Webb, D. J.: Notes on a 1-D Model of Continental Shelf Resonances, Research and Consultancy Report 85, National Oceanography Centre, Southampton, available at: http://eprints.soton.ac. uk/171197, 2011.

Wolanski, E.: Water Circulation in the Gulf of Carpentaria, J. Mar. Syst., 4, 401-420, 1993.

Wolanski, E., Ridd, P., and Inoue, M.: Currents through Torres Strait, J. Phys. Oceanogr., 18, 1535-1545, 1988.

Zahel, W. and Müller, M.: The computation of the free barotropic oscillations of a global ocean model including friction and loading effects, Ocean Dynam., 55, 137-161, 2005. 